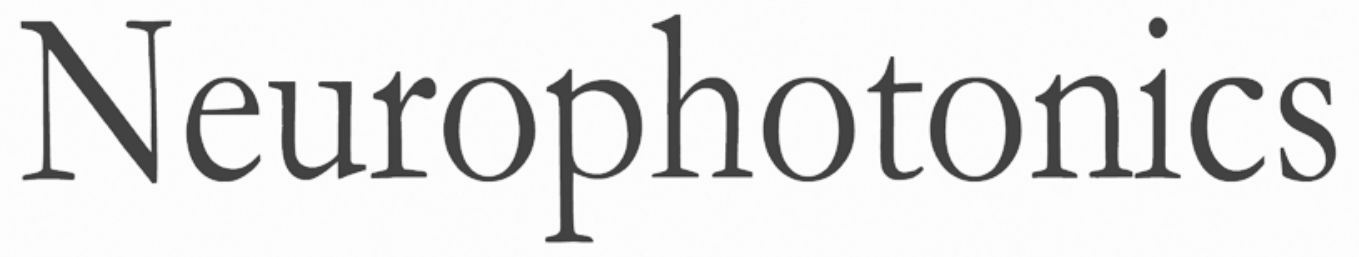

Review of quantitative phase-digital holographic microscopy: promising novel imaging technique to resolve neuronal network activity and identify cellular biomarkers of psychiatric disorders

Pierre Marquet

Christian Depeursinge

Pierre J. Magistretti 


\title{
Review of quantitative phase-digital holographic microscopy: promising novel imaging technique to resolve neuronal network activity and identify cellular biomarkers of psychiatric disorders
}

\author{
Pierre Marquet, ${ }^{a, b, \star}$ Christian Depeursinge, ${ }^{b, c}$ and Pierre J. Magistrettia, ${ }^{a, b}$ \\ ${ }^{a}$ Centre Hospitalier Universitaire Vaudois (CHUV), Centre de Neurosciences Psychiatriques, Département de Psychiatrie, Site de Cery, Prilly/ \\ Lausanne $\mathrm{CH}-1008$, Switzerland \\ ${ }^{\mathrm{b}}$ Brain Mind Institute, École Polytechnique Fédérale de Lausanne, Lausanne $\mathrm{CH}-1015$, Switzerland \\ 'King Abdullah University of Science and Technology (KAUST), Division of Biological and Environmental Sciences and Engineering, Thuwal \\ 23955-6900, Kingdom of Saudi Arabia
}

\begin{abstract}
Quantitative phase microscopy (QPM) has recently emerged as a new powerful quantitative imaging technique well suited to noninvasively explore a transparent specimen with a nanometric axial sensitivity. In this review, we expose the recent developments of quantitative phase-digital holographic microscopy (QP-DHM). Quantitative phase-digital holographic microscopy (QP-DHM) represents an important and efficient quantitative phase method to explore cell structure and dynamics. In a second part, the most relevant QPM applications in the field of cell biology are summarized. A particular emphasis is placed on the original biological information, which can be derived from the quantitative phase signal. In a third part, recent applications obtained, with QP$\mathrm{DHM}$ in the field of cellular neuroscience, namely the possibility to optically resolve neuronal network activity and spine dynamics, are presented. Furthermore, potential applications of QPM related to psychiatry through the identification of new and original cell biomarkers that, when combined with a range of other biomarkers, could significantly contribute to the determination of high risk developmental trajectories for psychiatric disorders, are discussed. () The Authors. Published by SPIE under a Creative Commons Attribution 3.0 Unported License. Distribution or reproduction of this work in whole or in part requires full attribution of the original publication, including its DOI. [DOI: 10.1117/1.NPh.1.2.020901]
\end{abstract}

Keywords: digital holographic microscopy; diffraction tomography; quantitative phase microscopy; cell imaging; absolute cell volume; co-transporters; transmembrane water movements; neuronal activities; glutamate; dendritic spines; early cell biomarkers; high-throughput screening; psychiatry disorders.

Paper 14050VR received May 20, 2014; revised manuscript received Aug. 8, 2014; accepted for publication Aug. 11, 2014; published online Sep. 22, 2014.

\section{Introduction}

Elucidating the mechanisms underlying physiological and pathophysiological cellular processes requires the possibility to quantitatively and noninvasively observe cell structure and dynamics down to cellular and subcellular levels. ${ }^{1}$ In addition, cell processes are usually multifaceted and cannot be efficiently grasped with techniques providing a view of a single aspect. Thus, the development of high resolution imaging approaches providing simultaneous measurements of multiple relevant biophysical cell parameters is a prerequisite to dissect complex cellular processes. Considering that most biological cells are transparent, i.e., they differ only slightly from their surroundings in terms of optical properties (including absorbance, reflectance, etc.), it is obvious that obtaining a comprehensive understanding of cellular physiological and pathophysiological processes with optical approaches remains a difficult challenge. Consequently, a wide range of optical approaches have been developed in cell biology. ${ }^{2}$ Specifically, the development of both new modes of contrast generation and new forms of high resolution microcopy techniques which allow us to break the so-called "diffraction limit" of resolution, which limits the

*Address all correspondence to: Pierre Marquet, E-mail: pierre.marquet@chuv .ch smallest observable details in the image to around $200-500 \mathrm{~nm}$, represent a very active area of research in the field of cell biology.

In contrast to fluorescence microscopy, which today represents a powerful and widely used cell imaging technique, ${ }^{4}$ contrast-generating modes, based on wavefront phase information, represent an intrinsic contrast of transparent specimens, which have already in the mid-20th century demonstrated their relevance for noninvasive visualization of cell structure, in particular because the Zernicke's invention of phase contrast $(\mathrm{PhC})^{5}$ and the DIC techniques developed by Smith $^{6}$ and Nomarski. Currently, PhC and Normarski's differential interference contrast (DIC) are widely used contrast-generating techniques available for high-resolution light microscopy. Without using any staining, $\mathrm{PhC}$ and DIC allow the visualization of transparent specimens making visible, in particular, the fine subcellular structural organization. Basically, these two noninvasive contrast-generating techniques are based on their capacity for transforming in detectable intensity modulation the minute relative phase shift that a transparent microscopic object induces either between the transmitted wave light and the undeviated background wave $(\mathrm{PhC})$ or between two orthogonally polarized transmitted waves (DIC).

In contrast to $\mathrm{PhC}$ and $\mathrm{DIC}$, the invention of interference microscopy with separate object and reference beams at the 
end of the 19th century provides a direct and quantitative measurement of the phase difference introduced by the observed object. Practically, due to the seminal work of Barer, interference microscopy applied to cell imaging has permitted the quantitative measurement of important biophysical cells parameters including dry mass ${ }^{8}$ and intracellular $\mathrm{RI}^{9}$. However, because of temporal phase shifting, interferometric techniques require very demanding and costly opto-mechanical designs and phase shifts are very sensitive to experimental artifacts and noise, only a few attempts to dynamically image live cells in biology have been reported. ${ }^{10,11}$

Nevertheless, scientific advances lowering the cost of lasers and data acquisition equipment as well as the development of computing facilities, the large spread of personal computers and digital signal processors have brought fundamentally new perspectives in the field of interferometry and holography. Consequently, during the last decades, numerous quantitative phase microscopy (QPM) techniques based on an interferometer or holographic approaches have been developed. These approaches are much simpler to implement than classical interference microscopy and provide reliable and quantitative phase mapping of the observed specimen, therefore, they have started to be efficiently used in the field of cell biology. ${ }^{12,13}$

In a first part of this review, we present the current state of the art of quantitative phase measurement based on digital holographic microscopy (DHM) with a special focus on the advantages provided by the possibility to propagate the whole object wave (amplitude and phase) diffracted by the observed specimen during the numerical reconstruction of the digitally recorded holograms. A paragraph describing quantitative phase tomographic approaches providing a full three-dimensional (3-D) image-in particular a 3-D RI distribution-of the observed specimen are also presented. In a second part, we briefly describe some relevant applications related to cell biology provided by different QPM techniques. Special attention is paid to the biological information provided by the quantitative phase signal (QPS) through the derivation of specific cell biophysical parameters. In the third part, we describe how quantitative phase (QP)-DHM and quantitative phase tomography (QPT), due to their capacity for quantitatively monitoring cell structure and dynamics through the measurements of different cell biophysical parameters, can begin to address specific questions in the field of cellular neuroscience and psychiatric research. Specifically, the possibility to noninvasively visualize spine dynamics and resolve neuronal network activity by performing a simultaneous multiple sites optical recording of transmembrane currents as well as identifying new early cell biomarkers of vulnerability, neuroprogession, and diagnosis of mental disorders are discussed. Finally, some perspectives related to the development of QPM and/or QPT in high-throughput multimodality approaches are presented.

\section{Principles of Quantitative Phase Measurements Based on Digital Holographic Microscopy}

\subsection{Digital Holography}

Holography techniques were developed by Gabor in 1948 with the aim of improving the detection of spatial resolution in the X-ray wavelength by exploiting its lensless imaging capabilities. This resulted in the possibility of generating an exact replica during the illumination of the recorded hologram (reconstruction process) with a specific magnification of the full object wavefront created by the observed specimen. ${ }^{14}$ The use of digital means in holography gradually occurred at the end of the 1960s, when Goodman used a vidicon detector to encode a hologram which could be reconstructed on a computer. ${ }^{15}$ However, the interest in digital holography rose with the availability of cheaper digital detectors and charge-coupled device cameras. ${ }^{16,17}$ The two practical main approaches to recover the object wave from the recorded hologram are namely temporal decoding, i.e., phase shifting and spatial decoding i.e., off-axis methods. Phase-shifting reconstruction methods are based on the combination of several frames, and enable the suppression of the zero order and one of the cross terms through temporal sampling. ${ }^{18,19}$ The most well-known phase-shifting algorithm, proposed by Yamaguchi, ${ }^{20}$ is based on the recording of four frames separated by a phase shift of a quarter of a wavelength. Various combinations of frames derived from interferometry have been considered ${ }^{19,21}$ and many different approaches have been developed to produce the phase shift including high precision piezo-electric transducers, which move a mirror in the reference wave or acousto-optics modulators using the light frequency shift, etc. One of the main issues in phase-shifting methods is the requirement of several frames for reconstruction in interferometric setups, which are commonly very sensitive to vibrations. Thus, it could be difficult to ensure stable phase shifts and an invariant sample state during acquisition. In addition, the requirements on the accuracy of the phase shifts are rather high in regard to displacements on the order of magnitude of hundreds of nanometers, implying the use of high-precision transducers. Consequently, several attempts were made to either reduce the required number of frames for reconstruction, which led to two-frame reconstruction, ${ }^{22,23}$ or to simultaneously enable the recoding of the various phaseshift frames, by employing, for example, multiplexing methods. ${ }^{24}$ On the other hand, more refined algorithms were developed to relax the accuracy requirements of phase-shift methods. ${ }^{25-27}$ It should also be mentioned that the development of a fast temporal phase-shifting procedure within the framework of tomography ${ }^{28}$ as well as approaches based on the spatial analysis of fringe pattern, ${ }^{29,30}$ sometimes referred as spatial phase shifting, has permitted to successfully reconstruct the object wave in an off-axis configuration. .1,32 $^{2}$

The second main approach for recovering the object wave is based on an off-axis configuration, so that the different diffraction terms encoded in the hologram (zero-order wave, real and virtual images) are propagating in different directions, enabling their separation for reconstruction. ${ }^{33}$ This configuration was the one employed for the first demonstration of a fully numerical recording and reconstruction holography. ${ }^{16,17}$

In practice, reconstruction methods based on an off-axis configuration usually rely on Fourier methods to filter one of the diffraction terms. This concept was first proposed by Takeda et al. ${ }^{34}$ in the context of interferometric topography. The method was later extended for smooth topographic measurements for phase recovery ${ }^{35}$ and generalized for use in DHM with amplitude and phase recovery. ${ }^{36}$ As discussed in the following paragraphs, the main characteristic of this approach is its capability for recovering the complex object wave through only one acquisition, thus greatly reducing the influence of vibrations. However, as the diffraction terms are spatially encoded in the 
hologram, this one shot capability potentially comes at the cost of the usable bandwidth. In addition, the frequency modulation, induced by the angle between the reference and the object wave, has to guarantee the separability of the information contained in the different diffraction terms that are encoded in the hologram while carrying a frequency compatible with the sampling capacity of the digital detectors.

\subsection{Quantitative Phase Digital Holographic Microscopy}

A digital holographic microscope ${ }^{37}$ comprises both an optical setup devoted to hologram formation and software specially developed to numerically process the digitized hologram. The hologram results from the interference of the object beam with a reference wave that can be kept separate from the object beam. The goal is to precisely estimate the propagated wavefront corresponding to either the virtual or the real image of the specimen. Different optical setups have been proposed to perform microscopy with holography. Some lensless setups have been proposed to image small fields ${ }^{38-40}$ by using strongly diverging wavefields, such as spherical waves emitted by punctual sources. Optical setups that make use of a microscope objective (MO) offer the largest numerical apertures. ${ }^{36,41}$ A typical DHM arrangement we have developed and used to explore transparent specimens including living cells, is the quantitative monitoring of the phase retardation of the transmitted wavefront [Fig. 1(a)]. ${ }^{41}$

Other configurations are possible depending on the targeted application, ${ }^{12,42}$ but will not be reviewed in detail here. An important issue is the need for a reference beam that is controllable in both in intensity and polarization, thus enabling one to improve contrast and signal. The holographic principle also permits other valuable concepts which particularly building on the possibility of superimposing several holograms. Holograms with several reference waves corresponding to several polarization states can be generated in order to analyze the birefringence properties of specimens including strained dielectrics or biological molecules. ${ }^{43,44}$ Reference waves corresponding to different wavelengths can also be generated, permitting the use of synthetic wavelengths from a single hologram. $^{45}$

As in other developments in digital holography, the hologram reconstruction method in DHM is based on the theory of diffraction. When an MO is used and the specimen can be maintained in the MO's focal plane, the image is formed at infinity. The hologram at infinity is simply the Fourier transform of the specimen. Inverse Fourier transformation straightforwardly provides the reconstructed wavefront in the focal plane of the MO. However, specimens are generally not fully contained in the focal plane of the MO, and thus a real and/or virtual image is formed at a finite distance from

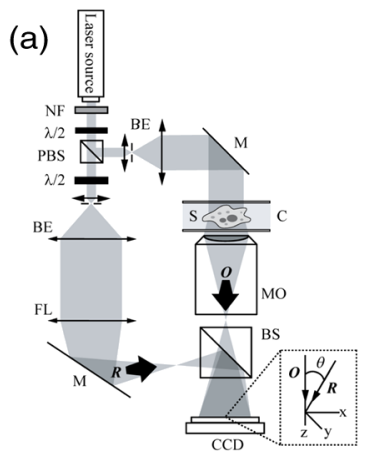

(c)

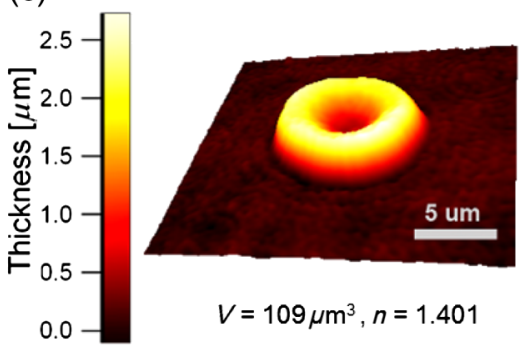

(b)

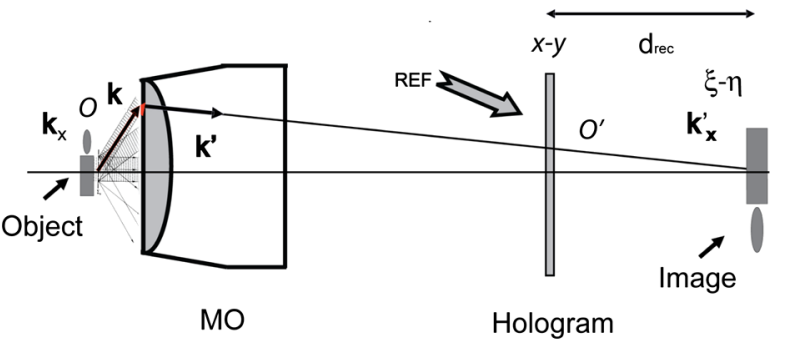

(d)

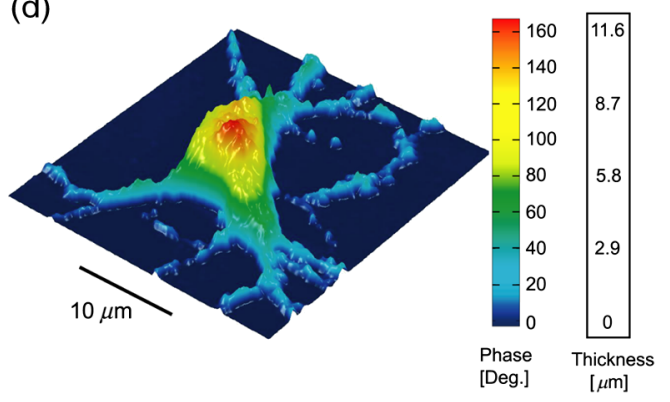

Fig. 1 (a) Transmission optical setup for QP-DHM (b) role of the microscope objective (MO): magnifying the image of the specimen but also, in terms of wavevectors, decreasing the lateral wavevector component $k_{x}$ or $y$ by a factor proportional to the magnification (M) of the MO, so that the hologram can be sampled by an electronic camera according to the Shannon's rules (c,d) three-dimensional (3-D) perspective images in false colors of a living human red blood cell (RBC) and a mouse cortical neuron in culture, respectively. Each pixel represents a quantitative measurement of the phase retardation or cellular optical path length (OPL) induced by the cell with a sensitivity corresponding to a few tens of nanometers. By taking into account the measured mean values of the RBC and neuronal cell body refractive indices (RIs) resulting from the decoupling procedure (cf. below), scales (at right), which relate OPL (deg) to morphology in the $z$-axis $(\mu \mathrm{m})$, can be constructed. Abbreviations: BS, beam splitter; CCD, camera; M1 and $\mathrm{M} 2$, mirrors; $\mathrm{MO}$, microscope objective; $\mathrm{O}$, object beam; $\mathrm{R}$, reference beam; RL, reference lens and $\mathrm{OC}$, object condenser; object or specimen. 
the MO [Fig. 1(b)]. Intercepting the wavefront at a finite distance from the specimen image gives rise to holograms in the Fresnel zone. Thus, the Huygens-Fresnel expression of diffraction can be used to calculate the propagation of the reconstructed wave. It should be noted that the MO introduced in the interferometer allows one to adapt the wavefield to the sampling capacity of the camera. Indeed, the lateral components of the wavevector $k_{x}$ or $y$ can be divided by the magnification factor $\mathrm{M}$ of the MO. Taking advantage of a full digital approach, a computer can perform the reconstruction by simulating the reference wave and multiplying it by the hologram intensity distribution. Then, the distribution of the wavefront in space is obtained by simulating the propagation of the wavefront reconstructed in the plane of the hologram. As previously mentioned, the wavefront gives rise to several propagated beams corresponding to the zero, +1 and -1 diffracted orders. When an MO is used, the latter two orders give rise to real and virtual images, respectively, that appear as twin images which are the reflection of one another on a virtual mirror that takes the place of the hologram plane.

The off-axis geometry introduces a spatial carrier frequency, and demodulation restores the full spatial frequency content of the wavefront. The main advantage of this approach is that all the information for reconstructing the complex wavefield comes from a single hologram. ${ }^{36}$ In microscopy, the full bandwidth of the beam delivered by the MO can be acquired without limitation. Scale adjustments can made to preserve the correct sampling of the MO's full bandwidth so that no loss of resolution is conceded. Finally, selection of a signal corresponding to a diffraction order of +1 in the Fourier domain of the hologram allows for the full restoration of the object wavefront transmitted by the MO. Therefore, reconstructing the wavefront in 3-D is simply done by propagating the wavefront generated in the hologram plane $x-y$ to the image plane $\xi-\eta$, which is situated at a distance $d_{\text {rec }}$. This could be efficiently achieved by computing the Fresnel transform of the wavefield. Thus, DHM brings quantitative data simultaneously derived from the amplitude and the phase of the complex reconstructed wavefront that is diffracted by the object. Our method requires the adjustment of several reconstruction parameters, which can be done using a computer-aided method we developed. Some image processing is also needed to improve the accuracy of the reconstructed phase. ${ }^{46}$ Moreover any type of aberrations can also be corrected during such a numerical reconstruction process. ${ }^{47,48}$ Using a high numerical aperture, submicron transverse resolution has been achieved to a 300-nm lateral resolution. Accuracies of approximately $0.1^{\circ}$ have been estimated for phase measurements. In reflection geometry, this corresponds to a vertical resolution of less than $1 \mathrm{~nm}$ at a wavelength of $632 \mathrm{~nm} .{ }^{49} \mathrm{In}$ transmission geometry, the resolution is limited to a few nanometers as far as living cells are concerned. It is also worthwhile to note that when the experimental configuration of the hologram recording requires distances of reconstruction for which the Fresnel approximation does not properly work, the propagation of the wavefront generated by the hologram can be efficiently performed with the angular spectrum approach. ${ }^{50,51}$ On the other hand, the characterization of the effect of noise on the formation of the hologram and on the reconstruction of the image results in an improved signal-to-noise ratio (SNR), using the coherent detection of scattered light. ${ }^{52,53}$ This improvement is often described as "coherent amplification" of the signal.

\subsection{Quantitative Phase Tomographic Microscopy: Full Three-Dimensional Imaging Technique}

In general, a single wavefront determination obtained from a single hologram is not sufficient to obtain a full 3-D image of an object. An exception concerns sparse media, similar to those found in particle image velocimetry (PIV), where each particle can be considered as an isolated punctual object and its position determined in 3-D by the methods of "in-line" holography. ${ }^{54,55}$ Thus, the combination of data reconstructed from several holograms, obtained at either several wavelengths (variable $k$-vector amplitude) or at several incidence angles (variable $k$-vector direction), is needed to achieve true 3-D imaging. Several articles report results obtained by changing the wavelength (variable $k$-vector amplitude) as shown by Marron. ${ }^{56}$ However, in this study, the range of the wavelength scan remained very small and consequently the resolution was weak. Arons et al. using "Fourier synthesis holography",57 have also discussed a similar approach. In digital holography, multiple wavelengths have been used to reconstruct 3-D structures. ${ }^{58}$ In the field of microscopy, such multiwavelength tomographic imaging techniques, based on reconstruction from multiple holograms, have yielded very accurate images of cells, erythrocytes in particular. ${ }^{59,60}$ An alternative, but somehow similar approach, is to use a wide bandwidth source and form a hologram in the plane where the mutual coherence between the object and reference wave is nonzero: this concept introduces coherence gating in the space domain. It has been proven to perform well. ${ }^{61,62}$ On the other hand, the variable $k$-vector direction approach more exactly meets the concept found in the literature under the name of "diffraction tomography" 63 for reconstruction of the scattering potential associated with the structure of the diffracting object. A diffracted wave can be collected and reconstructed from the holograms at various incidences. Practically, the phase and amplitude of the diffracted wave are directly reconstructed from the hologram and can be used to compute the scattering potential at every point of the specimen. From these data, the 3-D distribution of the RI can be established providing a 3-D tomographic image of the cell constituents. Tomography of cells based on DHM is new and original. In 2006, a first approach consisting of the rotation of the specimen, ${ }^{64,65}$ has been developed. It was demonstrated that the RI of the cell body could be measured in 3-D with a spatial resolution better than $3 \mu \mathrm{m}$ in all directions. Other approaches consisting of rotating the beam rather than the object have been proposed. ${ }^{28,66-69} \mathrm{Kou}^{70}$ and Sheppard ${ }^{71}$ have compared both approaches in their principles and shown consistent differences in the performance of each modality. Different reconstruction algorithms have been used to reconstruct objects from several holograms obtained at variable $k$-vector directions or amplitudes including projection ${ }^{28,64}$ and diffraction algorithms. ${ }^{72-74}$

\section{Cell Imaging with Quantitative Phase Microscopy}

Biological specimens, such as living cells and tissues, are usually phase objects, that is they are transparent and made visible most often by $\mathrm{PhC}$ as explained above. The phase signal originates in the RI difference generated by the presence of organic molecules in cells, including proteins, DNA, organelles, and nuclei. Consequently, QPM visualizes cells by quantitatively providing the phase retardation that they induce on the transmitted wavefront. ${ }^{41} 3$-D perspective images of such a phase retardation corresponding to a living human red blood cell and a 
mouse cortical neuron in culture are shown in Figs. 1(c) and $1(\mathrm{~d})$, respectively. This QPS signal is given by the following equation:

$\varphi=\frac{2 \pi}{\lambda}\left(\bar{n}_{c}-n_{m}\right) d$,

where $d$ is the cellular thickness, $\bar{n}_{c}$ is the intracellular RI averaged over the optical path length of optical rays crossing the specimen, and $n_{m}$ is the RI of the surrounding medium. The cellular quantitative phase contrast results from the fact that the intracellular RI is different from the RI of the perfusion solution $n_{m}$.

\subsection{Cell Counting, Classification Analysis, and Cytotoxic Screening Assay}

Practically, the QPS is particularly well suited to the development of algorithms, allowing automated cell counting, ${ }^{75-79}$ recognition, and classification. ${ }^{80-89}$ As far as red blood cells (RBCs) are concerned, QPM allows one to achieve a morphometry-based RBCs' analysis. Indeed, the intracellular content of mature nucleus-and organelle-free RBCs, which can be assimilated from an optical point of view to a homogeneous solution mainly containing hemoglobin, induces a quantitative phase shift directly proportional to the RBC thickness. ${ }^{78,85,87,88,90-96}$

Since the QPS is also sensitive to intracellular RI, it specifically provides some information about the intracellular content. Based on this information, interesting applications allowing discrimination between physiological and pathophysiological states have been achieved, particularly in the fields of assisted reproduction $^{86,97,98}$ and cancer research. ${ }^{99-102}$ In addition, in vitro label-free cytotoxicity screening assays based on QPDHM have been recently validated due to the flexibility offered by numerical reconstruction. ${ }^{103-106}$

\subsection{Exploration of Cell Movements and Dynamics}

QPM approaches as nonscanning techniques are usually well suited to monitor dynamic cell processes. Dynamic motions during the migrations of human dendritic cells ${ }^{107}$ and subcellular contraction of embryonic cardiomyocytes ${ }^{108,109}$ have been successfully measured. On the other hand, due to the numerical propagation of the reconstructed object wavefront, QP-DHM allowing, one to apply autofocusing ${ }^{32,110,111-114,115}$ and extended depth of focus ${ }^{116-122}$ has opened the possibility of efficiently monitoring cell migration in 3-D. ${ }^{110,112,123-130}$ In addition, these numerical possibilities offer an alternative to the shallow depth of field of conventional microscopy, which hampers any fast 3-D tracking of cells in their environment specifically when microfluidic devices are considered. ${ }^{131,132}$ Coherently, autofocusing and extended depth of focus abilities also facilitate the 3-D tracking of micro- or nano-particles, ${ }^{126,133-135}$ particularly in combination with approaches capable of second harmonic generation, ${ }^{136,137}$ the use of which is highly promising in medical fields including drug carrier, tumor detection and treatment, gene therapy.

\subsection{Dry Mass, Cell Growth, and Cell Cycle}

As described by Eq. (1), the measured quantitative phase shift induced by an observed cell on the transmitted light wavefront is proportional to the intracellular RI, which mainly depends on protein content. Therefore, this measure can be used to directly monitor protein production, owing to a relation established more than 50 years ago by Barer. ${ }^{8}$ Practically, the phase shift induced by a cell is related to its dry mass (DM) by the following equation (converted to the International System of Units):

$\mathrm{DM}=\frac{10 \lambda}{2 \pi \alpha} \int_{S_{c}} \Delta \varphi \mathrm{d} s=\frac{10 \lambda}{2 \pi \alpha} \Delta \bar{\varphi} S_{c}$,

where $\Delta \bar{\varphi}$ is the mean phase shift induced by the whole cell, $\lambda$ is the wavelength of the illuminating light source, $S_{c}$ is the projected cell surface, and $\alpha$ is a constant known as the specific refraction increment (in cubic meters per kilogram) and is related to the intracellular content. $\alpha$ is approximated by $1.8-2.1 \times 10^{-3} \mathrm{~m}^{3} \mathrm{Kg}^{-1}$, when considering a mixture of all the components of a typical cell.

Recently, several groups using various QPM techniques have begun to exploit this phase-DM relationship to study the dynamics of cell growth as well as to the characterization of the cell cycle. ${ }^{138-143}$ As far as RBCs are concerned, this relationship has also been explicitly and implicitly used to calculate hemoglobin content, which corresponds to the RBC DM. ${ }^{92,144-147}$

\subsection{Cell Membrane Fluctuations and Biomechanical Properties}

RBCs, while being carried throughout the vascular tree to deliver oxygen from the lungs to the tissues, are squeezed as they pass capillaries which are often smaller than the cell diameter. This ability can be attributed to the remarkable elastic properties of the membrane structure. As a consequence of these elastic properties, RBCs show cell membrane fluctuations (CMFs) at the nanometric scale, often called flickering. Owing to their high sensitivity allowing one to quantitatively measure RBC membrane fluctuations over the whole cell surface, different QPM techniques have shed new light on these CMFs by providing quantitative information about the biomechanical properties of the RBC membrane. ${ }^{145,148-153}$ We should also mention the integration of QPM with optical tweezers, which represents a very promising tool, especially with respect to monitoring trapped objects along the axial direction including nanoparticles, ${ }^{154,155}$ as well as manipulating and testing the biomechanical properties of cells). ${ }^{81,156-160}$

\subsection{Dynamics of Absolute Cell Volume and Transmembrane Water Movements}

Membranes of animal cells are highly permeable to water; therefore, movement of water across membranes is dictated in large part by osmotic pressure gradients. ${ }^{161,162}$ Any imbalance in intracellular and extracellular osmolarity is paralleled by water movements across cell membranes affecting the cell volume along with the concentration of intracellular compounds. ${ }^{163}$ However, even at constant extracellular osmolarity, the volume constancy of any mammalian cell is permanently challenged by the normal activity of the cells. ${ }^{164}$ Thus, the maintenance of a constant volume is critical for cell homeostasis and requires the continued operation of cell volume regulatory mechanisms. ${ }^{161}$ Water crosses cell membranes through several routes (simple diffusion through the lipid bilayer, transmembrane proteins such as specialized water channels, aquaporins, etc.). ${ }^{162}$ Consequently, the accurate measurement of both the absolute cell volume and water membrane permeability represents indices which can provide highly relevant information about the mechanisms involved in the cell volume regulatory processes. However, the noninvasive measurement of these two parameters 
at the single cell level is not a trivial issue. ${ }^{165}$ Below, we present how these parameters can be calculated from the QPS although, as indicated by Eq. (1), information concerning the intracellular content related to $\bar{n}_{c}$ is intrinsically mixed with morphological information relating to a thickness $d$. Practically, different strategies have been developed to separately measure cell morphology and RI. Some authors ${ }^{166,167}$ measured the intracellular RI by trapping cells between two cover slips separated by a known distance. However, this approach which prevents cell movement, precludes the possibility of exploring dynamic cellular processes, including cell volume regulation. Recently, spectroscopy phase microscopy approaches ${ }^{145,147,168}$ have addressed this limitation, at least as far as cells with high intrinsic dispersion properties are considered, including red blood cells (owing to presence of hemoglobin pigment). However, such spectroscopy approaches are only applicable to a very limited variety of cell types, most of which have intrinsic dispersion almost identical to that of water. We have developed another approach called the decoupling procedure, to separately measure the parameters $\bar{n}_{c}$ and $d$ from the phase signal $\varphi$, based on a modification of the extracellular RI, $n_{m}$. Basically, this method consists of performing a slight alteration of the extracellular RI $n_{m}$ and recording two holograms corresponding to the two different values of $n_{m}$, providing two quantitative phase images from which $\bar{n}_{c}$ and $d$ can be calculated. We have considered two different approaches to modify $n_{m}$ : the first approach requires sequentially perfusing a standard cell perfusion solution and a second solution with a different RI, but with the same osmolarity to record the two corresponding holograms at a single wavelength. ${ }^{169}$ Practically, this procedure has permitted us to quantitatively measure some highly relevant RBC parameters, including mean corpuscular volume and mean corpuscular hemoglobin concentration. ${ }^{92}$ However, owing to the solution exchange time, this approach precludes the possibility of monitoring dynamic changes of cell morphology and RI that occur during fast biological processes. To overcome these drawbacks, we have developed a second approach based on a dual-wavelength DHM (DW-DHM $)^{45}$ which exploits the dispersion of the extracellular medium, enhanced by the utilization of an extracellular dye, to achieve separate measurements of the intracellular RI and the absolute cell volume in real time. ${ }^{170}$ These approaches have been successfully applied to study the osmotic water membrane permeability $P_{f}$-representing the water volume flux per unit of time per unit of membrane surface for a given applied osmotic gradient—by monitoring cell volume changes while retaining the cell functionality. ${ }^{165}$ In addition, resulting from the linear relationship between the intracellular RI and the DM as well as from a DM balance equation, it has been possible to determine the RI of the transmembrane flux $n_{f} .{ }^{165}$ The high precision with which $n_{f}$ can be determined reveals if the transmembrane water flux is accompanied by solute transport and provides some important information about the underlying mechanisms involved in the transmembrane water movements.

\section{Resolving Neuronal Network Activity and Visualizing Spine Dynamics}

\subsection{Background}

Within the brain, water is distributed between blood, cerebrospinal fluid, and interstitial and intracellular compartments. Practically, water moves across these compartments following the differences in osmotic and hydrostatic pressures. At constant hydrostatic pressure gradients, water movements largely depend on the osmotic gradients, which are created by the concentration of osmolytes, including ions, amino acids, and derivatives, in particular, some neurotransmitters, in the extracellular and intracellular compartments. At the microscopic level, water transport is involved in cell volume regulation and in controlling the dimensions of the extracellular space (ECS). ${ }^{171,172}$ However, neuronal activity challenges the hemostasis of these intra and extra cellular compartments. Indeed, considering a low intracellular concentration of $\mathrm{Na}^{+}$, resulting from both a neuronal membrane with a low permeability to $\mathrm{Na}^{+}$and sodium-potassium transporters continuously pumping out $\mathrm{Na}^{+}$, if the $\mathrm{Na}^{+}$conductances suddenly increase it will cause $\mathrm{Na}^{+}$to rush into the neuronal cell. This movement of $\mathrm{Na}^{+}$ions along their concentration gradient is also enhanced by the transmembrane electrostatic gradient corresponding to a negative resting membrane potential $(-70 \mathrm{mV})$ mainly due to a large quantity of negatively charged intracellular proteins. This inward flow of $\mathrm{Na}^{+}$ions changes the electrochemical gradient, which in turn produces a further rise in the membrane potential called a depolarization. This mechanism is precisely what initiates the action potential. Otherwise, the $\mathrm{Na}^{+}$currents flowing inward at a point on the axon during an action potential spread out along the axon and depolarize the adjacent sections of its membrane. When actions potentials are conducted down an axon-their size remains undiminished as they travel - they finally induces a depolarization in a presynaptic terminal button, which is the end of axonal branches which generally face the postsynaptic membrane of the neuron that receives the message. The two membranes face each other across the synaptic cleft which is a gap of around 20-nm wide. Practically, when the depolarization reaches the terminal button, small vesicles containing molecules of the neurotransmitter and located just inside the presynaptic membrane, fuse with the membrane and then break down, spilling their contents into the synaptic cleft. Then, the neurotransmitter diffuses across the synaptic clef and by attaching postsynaptic membrane opens neurotransmitter-receptor ion channels, which permit the passage of specific ions into or out the cell, thus changing the local membrane potential of the postsynaptic neuron, which could, in turn, induces or inhibits the generation of action potentials. $^{173}$

Consequently, these transmembrane ions movements and neurotransmitter releases including glutamate, glycine, and GABA (gamma-aminobutyric acid) related to the neuronal activity are accompanied by transmembrane water movements for osmotic reasons. This results in the regulation of both the ECS and the cell volume being affected by the level of neural activity.

On the other hand, a distinct feature of nervous tissue is the intricate network of synaptic connections among neurons with diverse morphologies. Although initial connections are formed largely through molecular mechanisms, it has been clearly established that electrical activities influence not only the structure and efficacy of existing synapses, but can also contribute to the production of new synapses, in particular through changes in the size and shape of dendritic spines and the formation on new ones. Indeed, synapses can mainly occur in three places of the postsynpatic neuron: on the soma, on other axons, and on the smooth surface of dendrites-branched projections which compose most of the receptive surface of a neuron- or on dendritic spines-small membranous protrusions, with typical sizes of a few hundred nanometers, that stud the dendrites. The 
axodendritic synapses represent the largest proportion of the excitatory synapses in the mammalian brain and the glutamate is the most common excitatory neurotransmitter, inducing a depolarization of the postsynaptic neurons particularly by opening the (NMDA), the 2-amino-3-(3-hydroxy-5-methylisoxazole4-yl) propionate (AMPA), and the kainate ionotropic receptors, representing the three main classes of glutamate-receptor ion channels. All these abilities of synapses to strengthen, weaken or to be created over time in response to increases or decreases to the neuronal network activity, called synaptic plasticity, that have been intensively studied in recent years, have been significantly stimulated by the development of new optical methods, which have permitted the resolution of the morphology and the dynamics of dendritic spines. ${ }^{174}$ Furthermore, plasticity mechanisms affect information transfer on multiple time scales and levels of specificity and play a pivotal role in integrative function and learning. However, with a few exceptions, including activity-induced LTP and LTD (long-term potentiation and long-term depression) and some specific activity-dependent mechanisms that can modulate the density of dendritic spines onto which the majority of fast excitatory synaptic transmission operates, our understanding of how electrical activity affects the structure and function of neuronal networks is very limited. Within this framework, the development of techniques that provide a noninvasive resolution of both local neuronal network activity and dendritic spine dynamics is highly relevant. In light of this, we present how QP-DHM can start to originally address both of these issues due to the possibilities of high resolution and of monitoring cell volume and transmembrane water.

\subsection{Imaging Neuronal Activity by Measuring Transmembrane Water Movements with Quantitative Phase Digital Holographic Microscopy}

With respect to the study of neuronal activity, electrophysiological approaches, in particular patch-clamp techniques, have permitted major breakthroughs leading, in particular, to the discovery of LTP and LTD, by setting a voltage across the neuronal membrane and directly measuring the current flowing through a single ion channel. Basically, a patch clamp is the best standard for assessing ion channel function because it allows the discrimination of ionic currents in the femto-ampere (10-15 fA) range with microsecond time resolution. Practically, the most commonly used patch-clamp mode is the whole-cell mode. In short, in this configuration, an open tip thin glass micropipette, which contains both an ionic solution mimicking the intracellular solution and chlorided silver electrode connected to a highly sensitive electronic amplifier, is pressed against a cell membrane. A brief strong suction is applied to both assist in the formation of a high-resistance seal between the glass and the cell membrane and to disrupt the membrane patch. The interior of the pipette becomes continuous with the cytoplasm and a bath electrode is used to set the zero level. In whole-cell measurements, the two main configurations are the (1) voltage-clamp mode and the (2) current-clamp mode. In the voltage-clamp mode, the cell membrane potential is kept constant via a negative feedback loop injecting a compensating current into the cell that resembles the current that is flowing through the whole-cell membrane. Practically, the recording of this compensating current provides information about the whole-cell membrane conductance. In addition, the current- voltage relationships can be investigated since the membrane potential can be manipulated independently of ionic currents. In contrast, in the current-clamp mode, the injected current is kept constant and changes in the membrane potential can be measured. However, a patch clamp is still a highly invasive laborious process requiring precise micromanipulations and a high degree of operations, which generally impose the recording of voltages on a limited number of cells that form a neuronal network. Optical techniques, however, seem to be an ideal solution for measuring membrane potentials since they are relatively noninvasive and could work both at low and high magnifications. For instance, calcium indicators used in combination with high resolution two-photon microscopy allow the performing measurements of the spiking activity from hundreds to several thousand neurons in mammalian circuits while still keeping track of the activity of each neuron individually. ${ }^{175,176}$ However, calcium imaging has its shortcomings and cannot substitute for voltage imaging. ${ }^{176}$ Practically, voltage imaging methods have lagged behind calcium imaging due to important challenges related to physical constraints of the measurements themselves, including an electrical field highly located in the thin membrane region, an essentially two-dimension plasma membrane which cannot contain an arbitrary number of voltage chromophores without disrupting its properties, as well as the fact that the plasma membrane represents only a small proportion of the total membrane surface in the neuron on which the chromophores are attached. Finally, the relatively high speed of the electrical responses of mammalian neurons also represents a serious challenge for voltage measurements. Consequently, despite some promising perspectives, the different voltage imaging methods suffer from poor SNR and secondary side effects, and they have up to now fallen short of providing single-cell resolution when imaging the activity of neuronal populations. $^{176}$

Otherwise, it is well known that neuronal activity induces modifications of the intrinsic optical properties at the subcellular, ${ }^{177-180}$ cellular ${ }^{181,182}$ and tissue level. ${ }^{183-185}$ Consequently, we have attempted to establish an accurate relationship between QPS and the transmembrane current. Practically, we set out experiments combining electrophysiology in a whole-cell configuration and QP-DHM, performed in a well-established biological model involving $\mathrm{Cl}^{-}$conductance, a human embryonic kidney (HEK) cell line, and transfected to express the neuronal ligand-gated chloride ion channel called $\mathrm{GABA}_{\mathrm{A}}$, the acronym of its endogenous ligand, the gamma-aminobutyric acid which is an inhibitory neurotransmitter. These experiments have stressed the fact that a net ionic influx corresponds to a phase decrease due to the osmotic water entry while a net ionic efflux induces a phase increase reflecting the osmotic exit of water. Under the following two assumptions-(1) the $n_{c}$ value linearly depends on the concentration of the different intracellular components according to the seminal work of Davies and Wilkins ${ }^{186}$ and (2) the volume variations induced by the transmembrane water movements accompanying the ionic movements are proportional to the number of ions having passed through the membrane or channels-it has been possible to establish the following relation between the QP signal $\varphi(t)$ and transmembrane current $I(t)$

$I(t)=\frac{V_{0}}{\varepsilon} \frac{d}{d t}\left[\frac{\varphi_{0}}{\varphi(t)}\right]^{1 / s-r}-\frac{1}{\varepsilon} \frac{\mathrm{d} \Delta V(t)}{\mathrm{d} t}$, 
where $V_{0}$ is the cell volume measured at the beginning of the experiments at a time $t=t_{0}, \Delta V$ are the nonelectrogenic volume changes corresponding to any cell volume variation except those directly related to a transmembrane net current, $\varepsilon$ is a constant representing the volume variation associated with the net charge movement through the cell membrane $[\mathrm{ml} / \mathrm{C}], r$ is a parameter related to the cell deformation associated with the volume variation and the parameter $s$ which takes cell processes inducing volume variations or not but leading to intracellular RI changes by mechanisms other than dilution or concentration of the intracellular content by ions and water fluxes. ${ }^{187}$ Practically, $r \in[0,1]$ with $r=1 / 3$ and $r=1$ correspond to isotropic volume changes and piston-like cell movements in the $z$ direction, i.e., along the optical axis, respectively. Usually, the adhesion of cultured cells on a coverslip facilitates a movement along the $z$ axis resulting in $r \in[0.5,0.8]$ typically. In contrast, the case $s=$ 1 corresponds to a simple dilution or concentration of the cell content, while $s<1$ reflects modification of intracellular RI by processes mediating the release or cellular internalization of substrates, including protein release mechanisms or cotransport activation involved in the volume regulatory processes. Practically, the parameters $V_{0}, r$, and $s$ can be determined by the decoupling procedure. Consequently, two parameters remains to be determined, namely $\varepsilon$ and $\Delta V$, to calculate the current.

Experiments with HEK cells have validated Eq. (3) regarding $\mathrm{Cl}^{-}$currents mediated by GABA applications [Fig 2(a1)] with $\varepsilon_{\mathrm{GABA}}$ lies within the range of $90-110 \mu \mathrm{m}^{3} / \mathrm{nC}$, and have permitted us to construct a phase response as a function of the clamped membrane potential ( $\varphi / \mathrm{V}$ curve) allowing the determination of the equilibrium potential for $\mathrm{Cl}^{-}$with the same precision as an electrophysiological approach measuring the classical I/V curves [Fig. 2(a2)]. ${ }^{187}$ Practically, $\varepsilon_{\mathrm{GABA}}$ has been estimated for a membrane potential clamped at $-40 \mathrm{mV}$ by performing a least-squares fit to adjust the phase current (Pred. Curr.) derived from Eq. (3) with the measured current (electrophysiological recordings in a voltage-clamp mode). Using this $\varepsilon_{\mathrm{GABA}}$ determination, Pred. Curr. has been calculated for other values of the membrane potential by using Eq. (3) [Fig. 2(a1)].

Subsequently, Eq. (3) has been used to optically monitor neuronal network activity mediated by glutamate application, the main excitatory neurotransmitter in the brain released at $80 \%$ of the synapses. Practically, a multimodality microscope, QPDHM, and electrophysiology setup has been developed to study the early stage of neuronal responses induced by glutamate on primary culture of mouse cortical neurons. This study involving pharmacological experiments revealed that glutamate produces three distinct optical responses, predominantly mediated by NMDA receptors: biphasic (Biph.), reversible decrease (RD), and irreversible decrease (ID) responses [Fig. 2(b3)]. The shape and amplitude of the optical signal were not associated with a particular cellular phenotype, but reflected the physio-pathological status of neurons linked to the degree of NMDA activity. Thus, the "Biph.", "RD," and "ID" responses respectively indicated low-, a high-, and "excito-toxic" levels of NMDA activation. For these three distinct responses, a strong inward current associated with the early phase decrease, whose amplitude is proportional to the concentration of glutamate and to the duration of the application, has been measured. This inward current is consistent with glutamate-mediated activation of specific ionotropic receptors including the NMDA, AMPA, and the kainate ionotropic receptors, whose opening induces influxes of $\mathrm{Ca}^{2+}$ and $\mathrm{Na}^{+}$down their electrochemical gradient. Consistently, the phase decrease results from water entrance accompanying the influxes of $\mathrm{Ca}^{2+}$ and $\mathrm{Na}^{+}$for osmotic reasons. Moreover, furosemide and bumetanide, two inhibitors of sodium and/or potassium-coupled chloride movement, strongly modify the phase shift suggesting an involvement of two neuronal co-transporters, Na-K-Cl (NKCC1) and $\mathrm{K}-\mathrm{Cl}(\mathrm{KCC} 2)$ in the genesis of the optical signal. In addition, the phase responses can be decomposed into two components, a rapid one accompanying the glutamate-mediated current $\left(I_{\text {GLUT }}\right)$ - the phase decrease on Fig. 2(b1), and a slow one generally corresponding to a phase recovery while $I_{\mathrm{GLUT}}=0$. The phase recovery, which is much slower than the fast one, is likely to correspond to a nonelectrogenic neuronal volume regulation involving several mechanisms. Interestingly, the time course presented in Fig. 2(b1) shows that the water movements are not significantly delayed-at the tenth of second scale at least-relative to the recorded current. The measurements of $I_{\mathrm{GLUT}}$ as well as the corresponding intracellular RI and neuronal volume changes allowed us to estimate the parameter " $\varepsilon_{\mathrm{GLUT}}$ " $[\mathrm{mL} / \mathrm{C}]$. Practically, the values of $\varepsilon_{\text {GLUT }}$ lie within the range of $60-120 \mu \mathrm{m}^{3} / \mathrm{nC}$ equivalent to 340-620 water molecules transported per ion having crossed the membrane. Interestingly, the typical intracellular RI change induced by a glutamate pulse $(500 \mathrm{uM} 0.2 \mathrm{~s})$ is around $0.002-$ 0.003 . The associated neuronal swelling is around 100 femtoliter (fL) for a typical neuronal cell body of $1500 \mathrm{fL}$ corresponding to a $6-7 \%$ cell volume variation. However, these orders of magnitude correspond to an exogenous glutamate application during a few tenths of seconds. Physiological release of endogenous glutamate is likely to induce smaller water movements and intracellular RI changes. Finally, it is important to note that, although we do not yet have a comprehensive understanding of the underlying mechanisms inducing these phase responses, these consequent transmembrane movements of water accompanying the neuronal activity are in good agreement with the typical amount of water co-transported by various cotransports including NKCC $1 .^{188}$

Furthermore, information concerning transmembrane current obtained from electrophysiological recordings combined with QP-DHM signal allows us to pave the way for developing a simultaneous multiple sites optical recording of transmembrane currents capable of resolving local neuronal network activity. In addition, appropriate mathematical treatment of the optical signal affords the possibility to quantitatively determine the dynamics of the current triggered by the ionotropic receptor activity, making this technique amenable to use for pharmacological screenings of modulators developed for the management of human pathologies involving dysfunctions of specific ionotropic channel including chloride channels associated with cystic fibrosis. $^{189}$

\subsection{Three-Dimensional Visualization of Dendritic Spine Dynamics with Quantitative Phase Tomographic Microscopy}

As previously mentioned, dendritic spines are the postsynaptic components of most excitatory synapses in the mammalian brain and are the loci of structural and functional plasticity. In past decades, studies have revealed that the number and size of dendritic spines are regulated by a variety of gene products and environmental factors, underscoring the dynamic 
(a1)
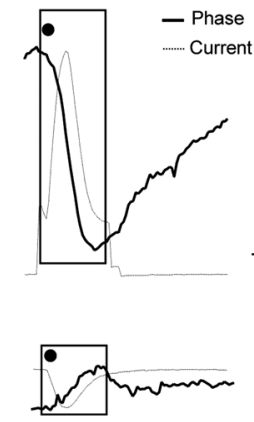

$-40 \mathrm{mV}$

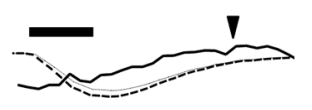

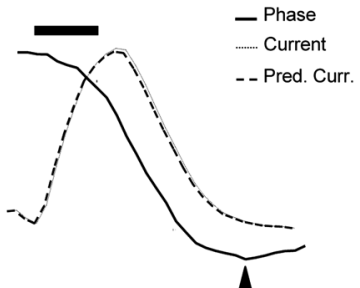

2.
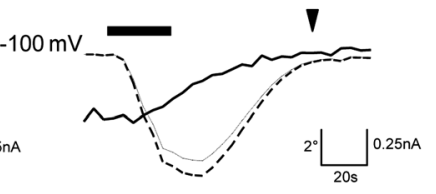

(a2)

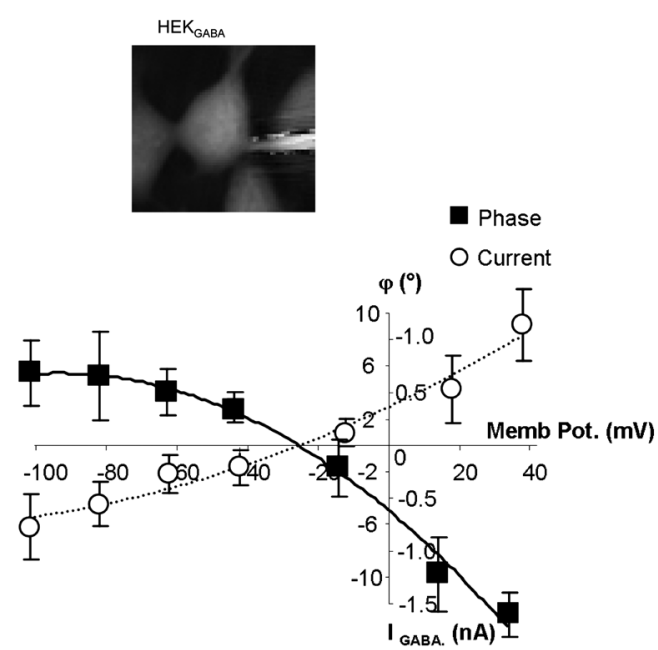

(b1)

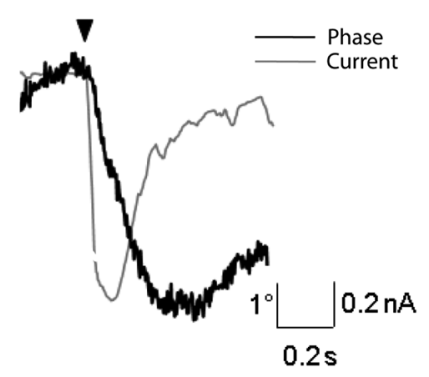

(b2)

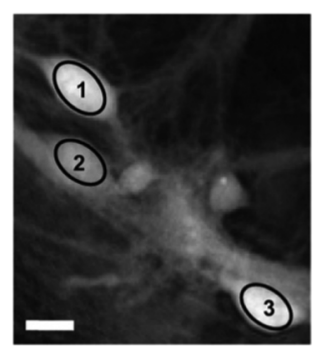

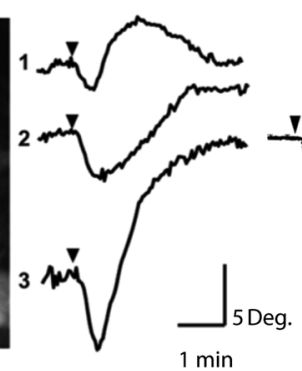

(b3)

Fig. 2 (A) HEK cells and GABA applications, (B) neurons in response to glutamate. (a1): (left) Example of three simultaneous traces of current (gray line) and phase shift (black line) recorded with $44 \mathrm{mM}$ of $\left[\mathrm{Cl}^{-}\right]_{\text {intrapip }}$ on the same HEK $\mathrm{GABA}$ after application of GABA (3 $\mu \mathrm{M} ; 30 \mathrm{~s}$; dot). At $-100 \mathrm{mV}$, application of GABA triggered an inward current concomitantly to an increase in the phase signal. Conversely, at $+40 \mathrm{mV}$, the same applications of GABA triggered an outward current accompanied by a decrease of the phase signal. Note that for $-40 \mathrm{mV}$ (close to the resting potential for $\mathrm{Cl}$ ), the current and the phase shift were very small. (a1) (right) Expansion of traces visualized in a1 (parts defined by rectangles). For each level of membrane potential, the peak of phase shift (indicated by the arrow head) was reached when the $I_{\text {GABA }}$ was terminated. According to Eq. (3) the phase signal can predict the current (Pred. Curr.: dashed line) superimposed on the recorded current: (a2) The $\varphi / V$ curve (full square and thick line) and the I/V curve (empty circle and thin line) obtained with GABA $(n=7)$ indicated an $\mathrm{E}_{\mathrm{CI}}$ of -26 and $-26 \mathrm{mV}$, respectively. (a2) inset representative quantitative phase image of patched a Hek cell. (b1): Local application of glutamate $(500 \mu \mathrm{M}, 200 \mathrm{~ms}$; arrow head) on a patched neuron induces a strong transient decrease of the phase signal associated to an inward current. Phase is expressed in degrees (b2) application of glutamate triggers three main types of phase shifts, Left, representative quantitative phase image of three cultured cortical neurons visualized in DHM. Ovals in the middle of the cells corresponded to the region of interest for the phase signal (scale bar, $10 \mu \mathrm{m})$. Right, application of glutamate $(30 \mu \mathrm{M}$, $30 \mathrm{~s}$; arrowhead) triggered different types of shapes of optical signals from these three recorded neurons. (b3), averaged traces of the three characteristic optical signals induced by glutamate application (30_M, $30 \mathrm{~s}$, arrowhead) and those recorded from 21-35 DIV neurons. These phase signals are classified into three categories: a biphasic response (Biph.; $n=137$ ), a reversible decrease of the phase signal (RD, $n=80$ ), and an irreversible decrease of the phase signal (ID, $n=35)$. All these experiments were performed at room temperature.

nature of spines and their importance to brain plasticity. Because dendritic spines are the key elements for information acquisition and retention, understanding how spines are formed and maintained in relation to neuronal network activity will likely provide fundamental insights into how the brain possesses the extraordinary capacity to learn and to remember. ${ }^{190}$ However, dendritic spines are tiny transparent processes whose visualization and resolution remain a challenge for optical approaches. However, the advent of two-photon (2P) excitation laser scanning microscopy, despite limitations inherent to the use of fluorescence, has greatly contributed to the understanding of dendritic spine function and plasticity. ${ }^{191,192}$ On the other hand, the diffraction tomography, which is particularly efficient when the wavelength of the irradiating beam is comparable to 
the dimensions of the diffracting parts of the object, represents a promising approach to dynamically and noninvasively resolve subcellular structures including dendritic spines. Indeed, appealing applications to cell biology have already been obtained and published. Practically, the investigation of the RI 3-D map has been published for pollen grains (Charrière, Marian et al. 2006), amoeba, ${ }^{65}$ human $\mathrm{RBCs}$ parasitized by plasmodium falciparum, ${ }^{193}$ human influenza virus infected cells, epithelial cheek cells, and granulocytes. ${ }^{194}$ Images of Hela cells at $0 \mathrm{deg}$ and up to 50 deg illumination incidences have been published ${ }^{195}$ and DIC has been derived from synthetic aperture phase contrast microscopy. Interestingly, an approach based on the rotation of the sample using a hollow optical fiber as a live-cell imaging chamber has permitted one to measure the RI 3-D maps of cell clusters. ${ }^{196}$ Recently, Cotte et al. firmly established the benefit of synthetic aperture elevated to quasi $2 \pi$ by pushing the occupation of the Fourier space up to a diameter equal to the diameter of the Ewald sphere. ${ }^{197}$ Super-resolution could be obtained and details as small as $100 \mathrm{~nm}$ could be observed. Moreover, dynamic images showing the detailed evolution of dendrite spines (Fig. 3) could be obtained.

\section{Quantitative Phase Digital Holographic Microscopy: Tool to Screen and Identify New Cell Biomarkers of Psychiatric Disorders}

\subsection{Background}

Psychiatric disorders represent major public health problems and, while significant improvements of the conditions of the patients have been achieved due to the discovery of neuroleptics and antidepressants, the available treatments remain largely insufficient and unsatisfactory. A major paradigm shift is needed to move the field forward. Advances in the field are impaired in large part due to the lack of proper tools to conduct adequate biological measurements that can guide diagnosis and treatment. This is particularly critical in light of growing evidence showing that early treatment of psychiatric disorders, already in their

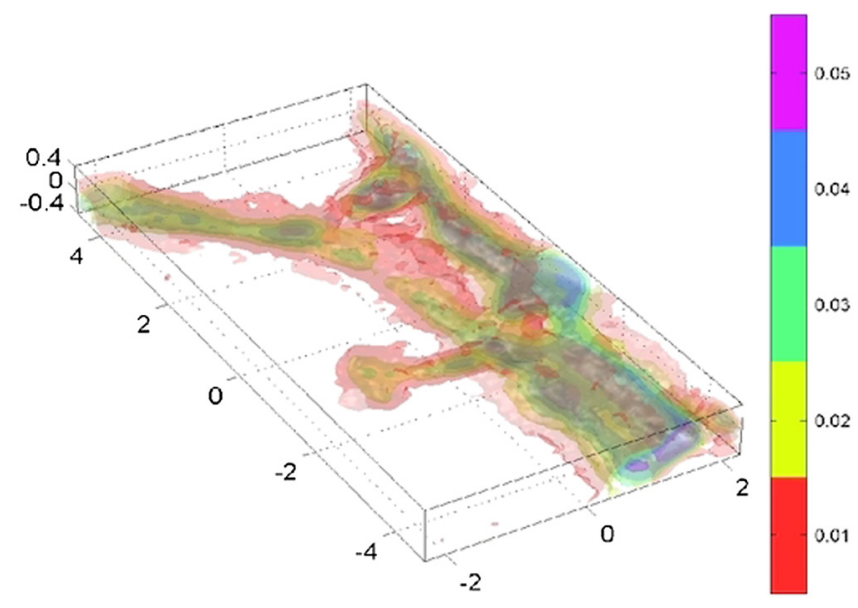

Fig. 3 Three-dimensional (3-D) representation of a tomographic image of a neuronal process obtained by synthetic aperture quantitative phase image. The details of protruding dendritic spines can be visualized. Scale is in $\mu \mathrm{m}$ and the color code represents the difference of refractive index (RI) [from 0.04 (red) to 0.16 (cyan)] compared with the extracellular medium (Yann Cotte). These experiments were performed at room temperature. prodromal phase and before the onset of debilitating symptoms, leads to significantly improved clinical outcomes. ${ }^{198}$ It is therefore important to identify in children of parents affected by the disease, biomarkers or endophenotypes indicating a high risk of developing the disease later in life However, to date there are insufficient data to support any of these biomarkers as vulnerability or diagnostic or prognostic factors, notably regarding bipolar disorders. ${ }^{199}$ In this field of research, the most relevant investigations are longitudinal studies conducted on cohorts consisting of patients with a specific psychiatric disorder and their children (high-risk children). Indeed, epidemiological studies, particularly in regard to mood disorders, ${ }^{200,201}$ have clearly shown that children of patients with a psychiatric disorder usually have a significantly higher risk of developing the disease. In addition, psychiatric disorders are frequently comorbid with other mental illnesses as well as endocrine, metabolic (e.g., diabetes mellitus and obesity) and cardiovascular diseases. Thus, they must be considered as a multisystem condition, and as a consequence, their underlying neurobiological bases are complex. Coherently, only studies combining several biomarkers have been successful in separating acute mood states (mania and depression) from controls ${ }^{202}$ and providing a high accuracy for the diagnosis of major depression. ${ }^{203}$ Consequently, the determination of high risk developmental trajectories for psychiatric disorders requires to identify and to longitudinally study, in high risk children, various sets of biomarkers very diverse in nature including, e.g., neuropsychological and neuroimaging (structural and functional MRI), as well as peripheral biomarkers or cellular biomarkers.

Practically, the study of cell biomarkers can be achieved from somatic cells (fibroblasts) obtained from patients as well as from high-risk children (skin biopsy). However, recent years have seen a renewed interest for the cell biomarkers due to the possibility, through induced pluripotent stem cells, to convert somatic cells (fibroblast) obtained from patients into neurons (grown in culture) with the genetic makeup necessary for the development of certain phenotypes of the disease. Although this approach currently has several limitations, ${ }^{204}$ it allows one to consider the particularly attractive hypothesis that some phenotypes are already found in vitro at the cell level or in a network of cells collected from patients or their offspring. ${ }^{205}$

Within this framework, a multimodality imaging approach developed around QP-DHM has the capacity to explore new, original optical cellular biomarkers down at the nanoscale that can then be put in contrast to other more conventional markers as mentioned above. Indeed, QP-DHM provides the unique monitoring of fine cell structure and dynamics due to its ability to yield quantitatively information on a wide variety of cellular parameters including volume, morphology, intracellular protein content, dry weight, nanoscale membrane fluctuations, membrane water permeability, transmembrane water movements, and volume regulation. The value of such an approach can be illustrated by a study we are conducting in a cohort of diabetic patients aimed at correlating energy distribution among vibrational eigenmodes of RBC membranes with the levels of glycosylated hemoglobin (Fig. 4).

Building on these results, we are conducting a study monitoring the RBC membrane eigenmode energy distribution in a sample of the prodromal phase of first-episode psychosis patients before initial treatment, aimed at identifying specific biomarkers for early detection and intervention. 
(a)

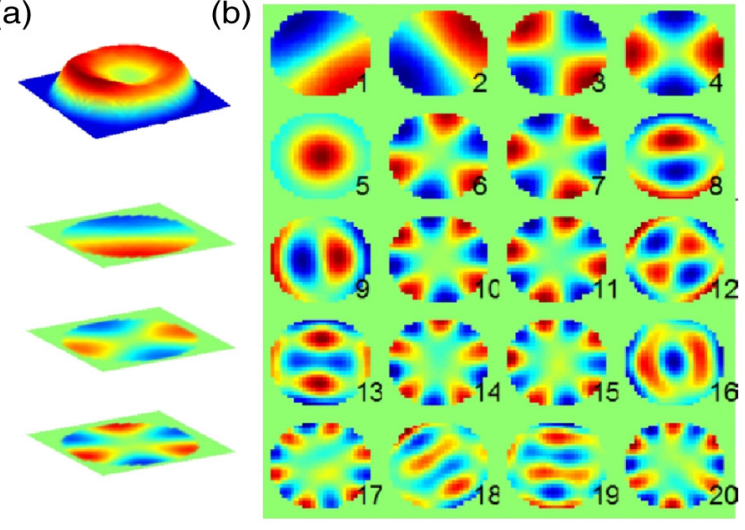

(c)

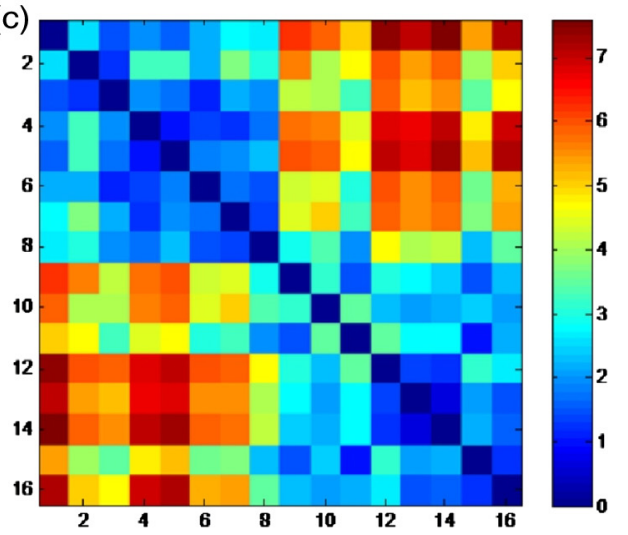

Fig. 4 (a) Quantitative phase image of a red blood cell (RBC) in a perspective color coded representation and projections of three principal component analysis (PCA) modes of the RBC cell membrane fluctuations. (b) Degeneracy free representation of 20 PCA modes, measured experimentally. (c) Each $t$-value $(\mathrm{i}, \mathrm{j})$ in the matrix indicates how similar the energy distributions of the PCA modes between subject $i$ and subject $j$ are. At lower $t$-values, the energy distribution becomes more similar. Healthy (1-8) and diabetic (9-16) samples can be discriminated.

\section{Perspectives}

As illustrated through these different applications, QPM and specifically DH-QPM because of the unique possibilities offered by the numerical reconstruction and propagation of the object wavefront including the extended depth of focus, a posteriori numerical autofocusing, allows one to quantitatively and noninvasively study cell structure and dynamics as well as the content at different scales of time ranging from a few milliseconds to several days. However, although QPS is highly sensitive and contains a wealth of information, its interpretation in terms of specific biological processes remains an issue. Practically, addressing this interpretation issue beyond the well-established calculation of the cell DM, involves the ability to calculate relevant biophysical cell parameters including absolute volume, membrane fluctuations at the nanoscale, biomechanical properties, transmembrane water permeability, current, and so on from the phase signal. Concretely, the derivation of these cell biophysical parameters often requires one to separately obtain cell morphology (thickness) and content (intracellular RI). Within this framework, the development of DHM-based optical diffraction tomography, providing a direct access to the 3-D map of the intracellular RI, could allow us to revisit this difficulty of interpretation. Future developments leading to the real time and high resolution 3-D maps of the intracellular RI could provide, in addition to the possibility of noninvasively resolving both neuronal network activity and dendritic spine dynamics as previously explained, valuable information about cytoachitecture and compartmentalization of cytoplasm, which plays a critical role in several fundamental cell mechanisms including proteins' synthesis. On the other hand, information about transmembrane water movements measured with DH-QPM allows us to pave the way for studying the activity of specific ionotropic receptors and/or co-transporters including nonelectrogenic ones as well as their modulation by specific pharmacological agents.

In addition, QP-DHM and QP-TM in multimodality approaches combining, e.g., fluorescence, ${ }^{206}$ spectroscopy, coherent anti-Stokes Raman scattering (CARS), second(SHG) and third-harmonic generation (THG), should allow simultaneous measurements of a large number of relevant and specific parameters yielding an extended cell profiling approach. Specifically, $\mathrm{SHG}^{207}$ and $\mathrm{THG}^{208}$ convey highly relevant information, particularly concerning membrane proteins and intracellular constituents. Due to the highly nonlinear character of these light generating processes, local properties of the cytoskeleton and membrane as well as 3-D cellular arrangements are obtainable by taking advantage of the remarkable penetration power of the SHG or, more particularly, the THG processes. Within this framework, it is worthwhile mentioning one of the first attempts to combine Raman information with quantitative phase information paving thus the way to simultaneously explore the cell's molecular composition and dynamics. ${ }^{209}$ Otherwise, used in conjunction with AFM, QP-DHM would provide an image of the local deformations, and, therefore, a more detailed and informative insight of biophysical properties of the cell. In addition, the use of free wandering particles would also precisely provide information on the nature of binding forces and properties of the macromolecules. This detection of multiple biophysical cell parameters could even be scaled into a high-throughput cellular screening assay ${ }^{105}$ allowing the concurrent monitoring of several cellular processes as well as their modulations as a function of the transcriptome and metabolome of different cell types derived from control patients as well as high-risk subjects. Thus, within the framework of longitudinal studies conducted on cohorts of patients and their children as previously mentioned, such high-throughput multimodality approaches would be highly promising to identify new and original early cell biomarkers or endophenotypes of mental disorders that will become invaluable tools for early diagnosis, improved stratification, a personalized medicine approach, and assessment of disease progression as well as of treatment outcome.

\section{Acknowledgments}

The work presented in this review is the result of a close collaboration between the microvision and microdiagnosis group (SCI/STI/CHD group) of EPFL with Florian Charrière, Jonas Kühn, Nicolas Pavillon, Etienne Shafer, Fatih Toy, Yann Cotte, the Laboratory of Neuroenergetics and Cellular Dynamics, Brain and Mind Institute of EPFL with Benjamin Rappaz, Pascal Jourdain, the Center for Psychiatric neuroscience, CHUV with Daniel Boss, Kaspar Rothefusser and the company Lyncée 
Tec SA, Lausanne (http://www.lynceetec.com). We thank the Swiss National Science Foundation (SNSF) Grant No. CR3213_132993 the National Center of competence in research Synapsy (http://www.nccr-synapsy.ch/) as well as the fondation de Préfargier.

\section{References}

1. V. Kumar, A. K. Abbas, and J. Aster, Robbins and Cotran Pathologic Basis of Disease, Elsevier Saunders, Philadelphia, PA (2014).

2. S. Inoué and R. K. Spring, Video Microscopy, The Fundamentals, Plenum, New York and London (1997).

3. R. Han et al., "Recent advances in super-resolution fluorescence imaging and its applications in biology," J. Genet. Genomics 40(12), 583595 (2013).

4. A. Cornea and P. M. Conn, Fluorescence Microscopy: SuperResolution and other Novel Techniques, Academic Press, USA (2014).

5. F. Zernike, "Phase contrast, a new method for the microsopic observation of transparent objects," Physica 9, 686-698 (1942).

6. F. H. Smith, "Microscopic interferometry," Research 8, 385-395 (1955).

7. G. Nomarski, “*'Dispositif Oculaire a Contraste De phase pour microscope," J. De Physique Et Le Radium 11(12), S9-S10 (1950).

8. R. Barer, "Interference microscopy and mass determination," Nature 169(4296), 366-367 (1952).

9. R. Barer, "Refractometry and interferometry of living cells," J. Opt. Soc. Am. 47(6), 545-556 (1957).

10. G. A. Dunn, "Transmitted-light interference microscopy: a technique born before its time," Proc. R. Microsc. Soc. 33, 189-196 (1998).

11. G. A. Dunn and D. Zicha, "Cell behaviour: adhesion and motility," Symp. Soc. Exp. Biol. 47, 91-106 (1993).

12. K. Lee et al., "Quantitative phase imaging techniques for the study of cell pathophysiology: from principles to applications," Sensors 13(4), 4170-4191 (2013).

13. P. Marquet, C. Depeursinge, and P. J. Magistretti, "Exploring neural cell dynamics with digital holographic microscopy," Annu. Rev. Biomed. Eng. 15, 407-431 (2013).

14. D. Gabor, "A new microscopic principle," Nature 161(4098), 777-778 (1948).

15. J. W. Goodman and R. W. Lawrence, "Digital image formation from electronically detected holograms," Appl. Phys. Lett. 11(3), $77-79$ (1967).

16. U. Schnars and W. Juptner, "Direct recording of holograms by a CCD target and numerical reconstruction," Appl. Opt. 33(2), 179-181 (1994).

17. O. Coquoz et al., "Performances of endoscopic holography with a multicore optical- fiber," Appl. Opt. 34(31), 7186-7193 (1995).

18. P. Rastogi, Holographic Interferometry: Principles and Methods, Springer-Verlag, New York (1994).

19. T. Kreis, Handbook of Holographic Interferometry: Optical and Digital Methods, Wiley-VCH Verlag GmbH \& Co. KGaA, Weinheim, FRG (2005).

20. I. Yamaguchi and T. Zhang, "Phase-shifting digital holography," Opt. Lett. 22(16), 1268-1270 (1997).

21. P. Carré, "Installation et utilisation du comparateur photoélectrique et interférentiel du Bureau International des Poids et Mesures," Metrologia 2(1), 13-33 (1966).

22. P. Guo and A. J. Devaney, "Digital microscopy using phase-shifting digital holography with two reference waves," Opt. Lett. 29(8), 857859 (2004).

23. J. P. Liu and T. C. Poon, "Two-step-only quadrature phase-shifting digital holography," Opt. Lett. 34(3), 250-252 (2009).

24. Y. Awatsuji, M. Sasada, and T. Kubota, "Parallel quasi-phase-shifting digital holography," Appl. Phys. Lett. 85(6), 1069-1071 (2004).

25. C. S. Guo et al., "Phase-shifting error and its elimination in phase-shifting digital holography," Opt. Lett. 27(19), 1687-1689 (2002).

26. Z. Wang and B. Han, "Advanced iterative algorithm for phase extraction of randomly phase-shifted interferograms," Opt. Lett. 29(14), 16711673 (2004).

27. X. F. Xu et al., "Simple direct extraction of unknown phase shift and wavefront reconstruction in generalized phase-shifting interferometry: algorithm and experiments," Opt. Lett. 33(8), 776-778 (2008).
28. W. Choi et al., "Tomographic phase microscopy," Nat. Methods 4(9), 717-719 (2007).

29. Y. Ichioka and M. Inuiya, "Direct phase detecting system," Appl. Opt. 11(7), 1507-1514 (1972).

30. L. Mertz, "Real-time fringe-pattern analysis," Appl. Opt. 22(10), 15351539 (1983).

31. D. Carl et al., "Parameter-optimized digital holographic microscope for high-resolution living-cell analysis," Appl. Opt. 43(36), 6536-6544 (2004).

32. M. Liebling, T. Blu, and M. Unser, "Complex-wave retrieval from a single off-axis hologram," J. Opt. Soc. Am. 21(3), 367-377 (2004).

33. E. N. Leith and J. Upatniek, "Reconstructed wavefronts and communication theory," J. Opt. Soc. Am. 52(10), 1123 (1962).

34. M. Takeda, H. Ina, and S. Kobayashi, "Fourier-transform method of fringe-pattern analysis for computer-based topography and interferometry," J. Opt. Soc. Am. 72(1), 156-160 (1982).

35. T. Kreis, "Digital holographic interference-phase measurement using the Fourier-transform method," J. Opt. Soc. Am. A 3(6), 847-855 (1986).

36. E. Cuche, P. Marquet, and C. Depeursinge, "Simultaneous amplitudecontrast and quantitative phase-contrast microscopy by numerical reconstruction of Fresnel off-axis holograms," Appl. Opt. 38(34), 6994-7001 (1999).

37. E. Cuche, F. Bevilacqua, and C. Depeursinge "Digital holography for quantitative phase-contrast imaging," Opt. Lett. 24(5), 291-293 (1999).

38. W. S. Haddad et al., "Fourier-transform holographic microscope," Appl. Opt. 31(24), 4973-4978 (1992).

39. Y. Takaki, H. Kawai, and H. Ohzu, "Hybrid holographic microscopy free of conjugate and zero-order images," Appl. Opt. 38(23), 49904996 (1999).

40. W. B. Xu et al., "Digital in-line holography for biological applications," Proc. Natl. Acad. Sci. U. S. A. 98(20), 11301-11305 (2001).

41. P. Marquet et al., "Digital holographic microscopy: a noninvasive contrast imaging technique allowing quantitative visualization of living cells with subwavelength axial accuracy," Opt. Lett. 30(5), 468-470 (2005).

42. G. Popescu, "Quantitative phase imaging of nanoscale cell structure and dynamics," Methods Cell Biol. 90, 87-115 (2008).

43. T. Colomb et al., "Polarization imaging by use of digital holography," Appl. Opt. 41(1), 27-37 (2002).

44. T. Colomb et al., "Polarization microscopy by use of digital holography: application to optical fiber birefringence measurements," Appl. Opt. 44(21), 4461-4469 (2005).

45. J. Kuhn et al., "Real-time dual-wavelength digital holographic microscopy with a single hologram acquisition," Opt. Express 15(12), 72317242 (2007).

46. E. Cuche, P. Marquet, and C. Depeursinge, "Spatial filtering for zeroorder and twin-image elimination in digital off-axis holography," Appl. Opt. 39(23), 4070-4075 (2000).

47. T. Colomb et al., "Automatic procedure for aberration compensation in digital holographic microscopy and application to specimen shape compensation," Appl. Opt. 45, 851-863 (2006).

48. T. Colomb et al., "Total aberrations compensation in digital holographic microscopy with a reference conjugated hologram," Opt. Express 14(10), 4300-4306 (2006).

49. J. Kuhn et al., "Axial sub-nanometer accuracy in digital holographic microscopy," Meas. Sci. Technol. 19(7), 074007 (2008).

50. B. Kemper et al., "Investigation of living pancreas tumor cells by digital holographic microscopy," J. Biomed. Opt. 11(3), 034005 (2006).

51. M. K. Kim, L. Yu, and C. J. Mann, "Interference techniques in digital holography," J. Opt. 8(7), S518-S523 (2006).

52. F. Charriere et al., "Shot-noise influence on the reconstructed phase image signal-to-noise ratio in digital holographic microscopy," Appl. Opt. 45(29), 7667-7673 (2006).

53. F. Charriere et al., "Influence of shot noise on phase measurement accuracy in digital holographic microscopy," Opt. Express 15(14), 88188831 (2007).

54. M. Liebling and M. Unser, "Autofocus for digital Fresnel holograms by use of a Fresnelet-sparsity criterion," J. Opt. Soc. Am. 21(12), 24242430 (2004). 
55. L. Dixon, F. C. Cheong, and D. G. Grier, "Holographic deconvolution microscopy for high-resolution particle tracking," Opt. Express 19(17), 16410-16417 (2011).

56. J. C. Marron and K. S. Schroeder, "Holographic laser-radar," Opt. Lett. 18(5), 385-387 (1993).

57. E. Arons and D. Dilworth, "Lensless imaging by spatial Fourier synthesis holography," Appl. Opt. 35(5), 777-781 (1996).

58. M. K. Kim, "Wavelength-scanning digital interference holography for optical section imaging," Opt. Lett. 24(23), 1693-1695 (1999).

59. F. Montfort et al., "Submicrometer optical tomography by multiplewavelength digital holographic microscopy," Appl. Opt. 45(32), 8209-8217 (2006).

60. J. Kuhn et al., "Submicrometer tomography of cells by multiple-wavelength digital holographic microscopy in reflection," Opt. Lett. 34(5), 653-655 (2009).

61. E. Cuche, P. Poscio, and C. Depeursinge, "Optical tomography by means of a numerical low-coherence holographic technique," J. Opt. 28(6), 260-264 (1997).

62. P. Massatsch et al., "Time-domain optical coherence tomography with digital holographic microscopy," Appl. Opt. 44(10), 1806-1812 (2005).

63. E. Wolf, "Three dimensional structure determination of semi-transparent object from holographic data," Opt. Commun. 1(4), 153-156 (1969).

64. F. Charriere et al., "Cell refractive index tomography by digital holographic microscopy," Opt. Lett. 31(2), 178-180 (2006).

65. F. Charriere et al., "Living specimen tomography by digital holographic microscopy: morphometry of testate amoeba," Opt. Express 14(16), 7005-7013 (2006).

66. M. Debailleul et al., "High-resolution three-dimensional tomographic diffractive microscopy of transparent inorganic and biological samples," Opt. Lett. 34(1), 79-81 (2009).

67. Y. J. Sung et al., "Optical diffraction tomography for high resolution live cell imaging," Opt. Express 17(1), 266-277 (2009).

68. Y. Cotte, M. F. Toy, and C. Depeursinge, "Beyond the lateral resolution limit by phase imaging," J. Biomed. Opt. 16(10), 106007 (2011).

69. M. Kim et al., "High-speed synthetic aperture microscopy for live cell imaging," Opt. Lett. 36(2), 148-150 (2011).

70. S. S. Kou and C. J. R. Sheppard, "Comparison of three dimensional transfer function analysis of alternative phase imaging methods," Proc. SPIE 6443, 64430Q (2007).

71. C. J. R. Sheppard and S. S. Kou, "3D Imaging with holographic tomography," in Proc. Int. Conf. on Advanced Phase Measurement Methods in Optics an Imaging, Vol. 1236, pp. 65-69, AIP (2010).

72. E. Wolf, "Three-dimensional structure determination of semi-transparent object from holographic data," Opt. Commun. 1(4), 153-156 (1969).

73. V. Lauer, "New approach to optical diffraction tomography yielding a vector equation of diffraction tomography and a novel tomographic microscope," J. Microsc-Oxford 205, 165-176 (2002).

74. A. Devaney and J. Cheng, "Optical diffraction tomography in an inhomogeneous background medium," Meas. Sci. Technol. 19(8), 085505 (2008).

75. J. H. Milgram and W. C. Li, "Computational reconstruction of images from holograms," Appl. Opt. 41(5), 853-864 (2002).

76. A. Molder et al., "Non-invasive, label-free cell counting and quantitative analysis of adherent cells using digital holography," J. Microsc. 232(2), 240-247 (2008).

77. S. Seo et al., "High-throughput lens-free blood analysis on a chip," Anal. Chem. 82(11), 4621-4627 (2010).

78. M. Mihailescu et al., "Automated imaging, identification, and counting of similar cells from digital hologram reconstructions," Appl. Opt. $\mathbf{5 0}$ (20), 3589-3597 (2011)

79. V. Bianco et al., "Imaging through scattering microfluidic channels by digital holography for information recovery in lab on chip," Opt. Express 21(20), 23985-23996 (2013).

80. I. Moon and B. Javidi, "Three-dimensional identification of stem cells by computational holographic imaging," J. R. Soc. Interface 4(13), 305313 (2007).

81. M. DaneshPanah et al., "3-D holographic imaging and trapping for noninvasive cell identification and tracking," J. Disp. Technol. 6(10), 490499 (2010).
82. B. Javidi, M. Daneshpanah, and I. Moon, "Three-dimensional holographic imaging for identification of biological micro/nanoorganisms," IEEE Photon. J. 2(2), 256-259 (2010).

83. I. Moon, F. Yi, and B. Javidi, "Automated three-dimensional microbial sensing and recognition using digital holography and statistical sampling," Sensors 10(9), 8437-8451 (2010).

84. A. Anand, V. K. Chhaniwal, and B. Javidi, "Imaging embryonic stem cell dynamics using quantitative 3-D digital holographic microscopy," IEEE Photon. J. 3(3), 546-554 (2011).

85. R. Liu et al., "Recognition and classification of red blood cells using digital holographic microscopy and data clustering with discriminant analysis," J. Opt. Soc. Am. 28(6), 1204-1210 (2011).

86. P. Memmolo et al., "Identification of bovine sperm head for morphometry analysis in quantitative phase-contrast holographic microscopy," Opt. Express 19(23), 23215-23226 (2011).

87. I. Moon et al., "Automated statistical quantification of three-dimensional morphology and mean corpuscular hemoglobin of multiple red blood cells," Opt. Express 20(9), 10295-10309 (2012).

88. F. Yi, C. G. Lee, and I. K. Moon, "Statistical analysis of 3D volume of red blood cells with different shapes via digital holographic microscopy," J. Opt. Soc. Korea 16(2), 115-120 (2012).

89. F. Yi et al., "Automated segmentation of multiple red blood cells with digital holographic microscopy," J. Biomed. Opt. 18(2), 026006 (2013).

90. G. Popescu et al., "Erythrocyte structure and dynamics quantified by Hilbert phase microscopy," J. Biomed. Opt. 10(6), 060503 (2005).

91. I. Bernhardt et al., "Application of digital holographic microscopy to investigate the sedimentation of intact red blood cells and their interaction with artificial surfaces," Bioelectrochemistry 73(2), 92-96 (2008).

92. B. Rappaz et al., "Comparative study of human erythrocytes by digital holographic microscopy, confocal microscopy, and impedance volume analyzer," Cytometry Part A 73A(10), 895-903 (2008).

93. M. Mir, K. Tangella, and G. Popescu, "Blood testing at the single cell level using quantitative phase and amplitude microscopy," Biomed. Opt. Express 2(12), 3259-3266 (2011).

94. D. S. Mehta and V. Srivastava, "Quantitative phase imaging of human red blood cells using phase-shifting white light interference microscopy with colour fringe analysis," Appl. Phys. Lett. 101(20), 203701 (2012).

95. N. T. Shaked et al., "Dynamic quantitative microscopy and nanoscopy of red blood cells in sickle cell disease," Proc. SPIE 8227, 822711 (2012).

96. I. Moon et al., "Automated quantitative analysis of 3D morphology and mean corpuscular hemoglobin in human red blood cells stored in different periods," Opt. Express 21(25), 30947-30957 (2013).

97. I. Crha et al., "Digital holographic microscopy in human sperm imaging," J. Assist. Reprod. Genet. 28(8), 725-729 (2011).

98. F. Merola et al., "Digital holography as a method for 3D imaging and estimating the biovolume of motile cells," Lab Chip 13(23), 4512-4516 (2013).

99. C. J. Mann et al., "High-resolution quantitative phase-contrast microscopy by digital holography," Opt. Express 13(22), 8693-8698 (2005).

100. H. Janeckova, P. Vesely, and R. Chmelik, "Proving tumour cells by acute nutritional/energy deprivation as a survival threat: a task for microscopy," Anticancer Res. 29(6), 2339-2345 (2009).

101. P. Wang et al., "Nanoscale nuclear architecture for cancer diagnosis by spatial-domain low-coherence quantitative phase microscopy," Proc. SPIE 7907, 790704 (2011).

102. P. Wang et al., "Nanoscale nuclear architecture for cancer diagnosis beyond pathology via spatial-domain low-coherence quantitative phase microscopy," J. Biomed. Opt. 15(6), 066028 (2010).

103. A. Bauwens et al., "Differential cytotoxic actions of Shiga toxin 1 and Shiga toxin 2 on microvascular and macrovascular endothelial cells," Thromb. Haemost. 105(3), 515-528 (2011).

104. N. Pavillon et al., "Early cell death detection with digital holographic microscopy," PLoS One 7(1), e30912 (2012).

105. J. Kuhn et al., "Label-free cytotoxicity screening assay by digital holographic microscopy," Assay Drug Dev. Technol. 11(2), 101-107 (2013).

106. B. Rappaz et al., "Digital holographic microscopy: a quantitative labelfree microscopy technique for phenotypic screening," Comb. Chem. High Throughput Screen 17(1), 80-88 (2014).

107. J. Garay et al., "Crosstalk between PKA and Epac regulates the phenotypic maturation and function of human dendritic cells," J. Immunol. 185(6), 3227-3238 (2010). 
108. A. K. Ellerbee, T. L. Creazzo, and J. A. Izatt, "Investigating nanoscale cellular dynamics with cross-sectional spectral domain phase microscopy," Opt. Express 15(13), 8115-8124 (2007).

109. R. Ansari et al., "Quantitative analysis of cardiomyocyte dynamics with optical coherence phase microscopy," Proc. SPIE 8213, 821338 (2012)

110. Y. S. Choi and S. J. Lee, "Three-dimensional volumetric measurement of red blood cell motion using digital holographic microscopy," Appl. Opt. 48(16), 2983-2990 (2009).

111. T. Kim and T. C. Poon, "Autofocusing in optical scanning holography," Appl. Opt. 48(34), H153-H159 (2009).

112. P. Langehanenberg et al., "Automated three-dimensional tracking of living cells by digital holographic microscopy," J. Biomed. Opt. 14(1), 014018 (2009).

113. H. Y. Wang et al., "photonics and optoelectronics," in SOPO Symposium on Photonics and Optoelectronics, pp. 1-4, IEEE (2009).

114. J. Zhao et al., "Autofocusing on pure phase object for living cell imaging in lensless Fourier transform digital holography," Proc. SPIE 7790, 779017 (2010).

115. M. F. Toy et al., "Digital holographic microscopy for the cytomorphological imaging of cells under zero gravity," Proc. SPIE 8227, 82270S (2012).

116. P. Ferraro et al., "3-D imaging with large focus extension by a coherent optical microscope," in Information Optics, G. Cristobal, B. Javidi, and S. Vallmitjana, Eds., 860, pp. 52-62 (2006).

117. P. Ferraro et al., "Extended focused image in microscopy by digital holography," Opt. Express 13(18), 6738-6749 (2005).

118. M. Antkowiak et al., "Accurate three-dimensional detection of microparticles by means of digital holographic microscopy," Proc. SPIE 6995, 699514 (2008).

119. C. McElhinney, B. Hennelly, and T. Naughton, "Extended focused imaging for digital holograms of macroscopic three-dimensional objects," Appl. Opt. 47(19), D71-D79 (2008).

120. I. Bergoend et al., "Depth-of-field extension and 3-D reconstruction in digital holographic microscopy," Proc. SPIE 7390, 73901C (2009).

121. T. Colomb et al., "Extended depth-of-focus by digital holographic microscopy," Opt. Lett. 35(11), 1840-1842 (2010).

122. W. Q. Pan, "Multiplane imaging and depth-of-focus extending in digital holography by a single-shot digital hologram," Opt. Commun. 286, 117-122 (2013).

123. C. J. Mann, L. F. Yu, and M. K. Kim, "Movies of cellular and subcellular motion by digital holographic microscopy," Biomed. Eng. Online 5, 21 (2006).

124. M. DaneshPanah and B. Javidi, "Tracking biological microorganisms in sequence of 3D holographic microscopy images," Opt. Express 15(17), 10761-10766 (2007).

125. H. Y. Sun et al., "Visualization of fast-moving cells in vivo using digital holographic video microscopy," J. Biomed. Opt. 13(1), 014007 (2008).

126. Y. Bae et al., "Three-dimensional single particle tracking using off-axis digital holographic microscopy," Proc. SPIE 7574, 757408 (2010).

127. B. Javidi, I. Moon, and M. Daneshpanaha, "Detection, identification and tracking of biological micro/nano organisms by computational 3D optical imaging," Proc. SPIE 7759, 77590R (2010).

128. M. Bohm et al., "PACE4, a member of the prohormone convertase family, mediates increased proliferation, migration and invasiveness of melanoma cells in vitro and enhanced subcutaneous tumor growth in vivo," J. Invest. Dermatol. 131, S108-S108 (2011).

129. P. Memmolo et al., "On the holographic 3D tracking of in vitro cells characterized by a highly-morphological change," Opt. Express 20 (27), 28485-28493 (2012).

130. F. Merola et al., "Simultaneous optical manipulation, 3-D tracking, and imaging of micro-objects by digital holography in microfluidics," IEEE Photon. J. 4(2), 451-454 (2012).

131. L. Miccio et al., "Detection and visualization improvement of spermatozoa cells by digital holography," Proc. SPIE 8089, 80890C (2011).

132. M. Paturzo et al., "Microscopy imaging and quantitative phase contrast mapping in turbid microfluidic channels by digital holography," Lab Chip 12(17), 3073-3076 (2012).

133. W. Xu et al., "Tracking particles in four dimensions with in-line holographic microscopy," Opt. Lett. 28(3), 164-166 (2003).

134. M. Antkowiak et al., "Extended focused imaging of a microparticle field with digital holographic microscopy," Opt. Lett. 33(14), 16261628 (2008).
135. N. Warnasooriya et al., "Imaging gold nanoparticles in living cell environments using heterodyne digital holographic microscopy," Opt. Express 18(4), 3264-3273 (2010).

136. C. L. Hsieh et al., "Three-dimensional harmonic holographic microcopy using nanoparticles as probes for cell imaging," Opt. Express 17(4), 2880-2891 (2009).

137. E. Shaffer and C. Depeursinge, "Digital holography for second harmonic microscopy: application to 3D-tracking of nanoparticles," Proc. SPIE 7715, 771505 (2010).

138. D. Zicha and G. A. Dunn, "An image-processing system for cell behavior studies in subconfluent cultures," J. Microsc-Oxford 179, 11-21 (1995).

139. G. Popescu et al., "Imaging red blood cell dynamics by quantitative phase microscopy," Blood Cells Mol. Dis. 41(1), 10-16 (2008).

140. B. Rappaz et al., "Noninvasive characterization of the fission yeast cell cycle by monitoring dry mass with digital holographic microscopy," $J$. Biomed. Opt. 14(3), 034049 (2009).

141. B. Kemper et al., "Label-free quantitative cell division monitoring of endothelial cells by digital holographic microscopy," J. Biomed. Opt. 15(3), 036009 (2010).

142. M. Mir et al., "Optical measurement of cycle-dependent cell growth," Proc. Natl. Acad. Sci. U. S. A. 108(32), 13124-13129 (2011).

143. H. Byun et al., "Optical measurement of biomechanical properties of individual erythrocytes from a sickle cell patient," Acta Biomater. 8 (11), 4130-4138 (2012).

144. A. I. Yusipovich et al., "Laser interference microscopy in erythrocyte study," J. Appl. Phys. 105(10), 102037 (2009).

145. Y. Jang, J. Jang, and Y. Park, "Dynamic spectroscopic phase microscopy for quantifying hemoglobin concentration and dynamic membrane fluctuation in red blood cells," Opt. Express 20(9), 96739681 (2012).

146. H. Pham et al., "Spectroscopic diffraction phase microscopy," Opt. Lett. 37(16), 3438-3440 (2012).

147. M. Rinehart, Y. Z. Zhu, and A. Wax, "Quantitative phase spectroscopy," Biomed. Opt. Express 3(5), 958-965 (2012).

148. G. Popescu et al., "Optical imaging of cell mass and growth dynamics," Am. J. Physiol. Cell Physiol. 295(2), C538-C544 (2008).

149. B. Rappaz et al., "Spatial analysis of erythrocyte membrane fluctuations by digital holographic microscopy," Blood Cells Mol. Dis. 42(3), 228-232 (2009).

150. Y. K. Park et al., "Metabolic remodeling of the human red blood cell membrane," Proc. Natl. Acad. Sci. U. S. A. 107(4): 1289-1294 (2010).

151. S. Lee et al., "Detrended fluctuation analysis of membrane flickering in discocyte and spherocyte red blood cells using quantitative phase microscopy," J. Biomed. Opt. 16(7), 076009 (2011).

152. Y. Park et al., "Measurement of the nonlinear elasticity of red blood cell membranes," Phys. Rev. E 83(5), 051925 (2011).

153. D. Boss et al., "Spatially-resolved eigenmode decomposition of red blood cells membrane fluctuations questions the role of ATP in flickering," PLoS One 7(8), e40667 (2012).

154. T. Higuchi et al., "Three-dimensional positioning of optically trapped nanoparticles," Appl. Opt. 50(34), H183-H188 (2011).

155. Y. Hayasaki and A. Sato, "Three-dimensional position measurement of a gold nanoparticle trapped in optical tweezers using in-line low-coherence digital holography," in Proc. 2012 11th Euro-American Workshop on Information Optics (Wio), IEEE, Québec (2012).

156. J. M. A. Mauritz et al., "Biophotonic techniques for the study of malaria-infected red blood cells," Med. Biol. Eng. Comput. 48(10), 1055-1063 (2010).

157. N. Cardenas, L. F. Yu, and S. K. Mohanty, "Probing orientation and rotation of red blood cells in optical tweezers by digital holographic microscopy," Proc. SPIE 7906, 790613 (2011).

158. N. Cardenas, L. F. Yu, and S. K. Mohanty, "Stretching of red blood cells by optical tweezers quantified by digital holographic microscopy," Proc. SPIE 7897, 78971J (2011).

159. M. Esseling et al., "Multimodal biophotonic workstation for live cell analysis," J. Biophotonics 5(1), 9-13 (2012).

160. B. Kemper et al., "Towards 3D modelling and imaging of infection scenarios at the single cell level using holographic optical tweezers and digital holographic microscopy," J. Biophotonics 6(3), 260-266 (2013). 
161. B. Friedrich, I. Matskevich, and F. Lang, "Cell volume regulatory mechanisms," Contrib. Nephrol. 152, 1-8 (2006).

162. E. K. Hoffmann, I. H. Lambert, and S. F. Pedersen, "Physiology of cell volume regulation in vertebrates," Physiol. Rev. 89(1), 193-277 (2009).

163. H. Pasantes-Morales and S. Cruz-Rangel, "Brain volume regulation: osmolytes and aquaporin perspectives," Neuroscience 168(4), 871884 (2010).

164. K. Strange, "Cellular volume homeostasis," Adv. Physiol. Educ. 28(4), $155-159$ (2004).

165. D. Boss et al., "Measurement of absolute cell volume, osmotic membrane water permeability, and refractive index of transmembrane water and solute flux by digital holographic microscopy," J. Biomed. Opt. 18(3), 036007 (2013).

166. N. Lue et al., "Live cell refractometry using microfluidic devices," Opt. Lett. 31(18), 2759-2761 (2006).

167. B. Kemper et al., "Integral refractive index determination of living suspension cells by multifocus digital holographic phase contrast microscopy," J. Biomed. Opt. 12(5), 054009 (2007).

168. Y. Park et al., "Spectroscopic phase microscopy for quantifying hemoglobin concentrations in intact red blood cells," Opt. Lett. 34(23), 3668-3670 (2009).

169. B. Rappaz et al., "Measurement of the integral refractive index and dynamic cell morphometry of living cells with digital holographic microscopy," Opt. Express 13(23), 9361-9373 (2005).

170. B. Rappaz et al., "Simultaneous cell morphometry and refractive index measurement with dual-wavelength digital holographic microscopy and dye-enhanced dispersion of perfusion medium," Opt. Lett. 33(7), 744-746 (2008).

171. E. Sykovà et al., "The relationship between changes in intrinsic optical signals and cell swelling in rat spinal cord slices," Neuroimage 18, 214-230 (2003).

172. E. A. Nagelhus, T. M. Mathiisen, and O. P. Ottersen, "Aquaporin-4 in the central nervous system: cellular and subcellular distribution and coexpression with KIR4.1," Neuroscience 129(4), 905-913 (2004).

173. N. Carlson, Physiology of Behavior, Pearson, Harlow (2014).

174. Z. Padamsey and N. J. Emptage, "Imaging synaptic plasticity," Mol Brain 4, 36 (2011).

175. R. Cossart, D. Aronov, and R. Yuste, "Attractor dynamics of network UP states in the neocortex," Nature 423(6937), 283-288 (2003).

176. D. S. Peterka, H. Takahashi, and R. Yuste, "Imaging voltage in neurons," Neuron 69(1), 9-21 (2011).

177. D. K. Hill and R. D. Keynes, "Opacity changes in stimulated nerve," J. Physiol. 108(3), 278-281 (1949).

178. I. Tasaki et al., "Changes in fluorescence, turbidity, and birefringence associated with nerve excitation," Proc. Natl. Acad. Sci. U. S. A. 61(3), 883-888 (1968).

179. L. B. Cohen, "Changes in neuron structure during action potential propagation and synaptic transmission," Physiol. Rev. 53(2), 373418 (1973).

180. K. M. Carter, J. S. George, and D. M. Rector, "Simultaneous birefringence and scattered light measurements reveal anatomical features in isolated crustacean nerve," J. Neurosci. Methods 135(1-2), 9-16 (2004).

181. R. A. Stepnoski et al., "Noninvasive detection of changes in membrane potential in cultured neurons by light scattering," Proc. Natl. Acad. Sci. U. S. A. 88(21), 9382-9386 (1991).

182. I. Tasaki and P. M. Byrne, "Rapid structural changes in nerve fibers evoked by electric current pulses," Biochem. Biophys. Res. Commun. 188(2), 559-564 (1992).

183. B. A. MacVicar and D. Hochman, "Imaging of synaptically evoked intrinsic optical signals in hippocampal slices," J. Neurosci. 11(5), 1458-1469 (1991).

184. R. D. Andrew, J. R. Adams, and T. M. Polischuk, "Imaging NMDAand kainate-induced intrinsic optical signals from the hippocampal slice," J. Neurophysiol. 76(4), 2707-2717 (1996).

185. K. Holthoff and O. W. Witte, "Intrinsic optical signals in rat neocortical slices measured with near-infrared dark-field microscopy reveal changes in extracellular space," J. Neurosci. 16(8), 2740-2749 (1996).

186. H. G. Davies and M. H. F. Wilkins, "Interference microscopy and mass determination," Nature 169(4300), 541 (1952).
187. P. Jourdain et al., "Simultaneous optical recording in multiple cells by digital holographic microscopy of chloride current associated to activation of the ligand-gated chloride channel GABA(A) receptor," PLoS One 7(12), e51041 (2012).

188. S. Hamann et al., "Water permeability of $\mathrm{Na}^{+}-\mathrm{K}^{+}-2 \mathrm{Cl}^{-}$cotransporters in mammalian epithelial cells," J. Physiol. 568(1), 123-135 (2005).

189. P. Jourdain et al., "The human CFTR protein expressed in CHO cells activates aquaporin-3 in a cAMP-dependent pathway: study by digital holographic microscopy," J. Cell Sci. 127(3), 546-556 (2014).

190. D. H. Bhatt, S. Zhang, and W. B. Gan, "Dendritic spine dynamics," Annu. Rev. Physiol. 71, 261-282 (2009).

191. K. Svoboda and R. Yasuda, "Principles of two-photon excitation microscopy and its applications to neuroscience," Neuron 50(6), 823-839 (2006).

192. K. F. C. Lee, C. Soares, and J. C. Beique, "Examining form and function of dendritic spines," Neural Plast. 2012, 704103 (2012).

193. Y. Park et al., "Static and dynamic light scattering of healthy and malaria-parasite invaded red blood cells," J. Biomed. Opt. 15(2), 020506 (2010).

194. B. Simon et al., "High-resolution tomographic diffractive microscopy of biological samples," J. Biophotonics 3(7), 462-467 (2010).

195. M. Kim et al., "Three-dimensional differential interference contrast microscopy using synthetic aperture imaging," J. Biomed. Opt. 17(2), 026003 (2012).

196. A. Kus et al., "Tomographic phase microscopy of living three-dimensional cell cultures," J. Biomed. Opt. 19(4), 046009 (2014).

197. Y. Cotte et al., "Marker-free phase nanoscopy (vol 7, pg 113, 2013)," Nat. Photonics 7(5), 418-418 (2013).

198. M. Berk et al., "From neuroprogression to neuroprotection: implications for clinical care," Med. J. Aust. 193(4 Suppl), S36-S40 (2010).

199. J. L. Luby and N. Navsaria, "Pediatric bipolar disorder: evidence for prodromal states and early markers," J. Child Psychol. Psychiatry 51(4), 459-471 (2010).

200. C. Vandeleur et al., "Mental disorders in offspring of parents with bipolar and major depressive disorders," Bipolar Disord. 14(6), 641-653 (2012).

201. E. Mesman et al., "The Dutch bipolar offspring study: 12-year followup," Am. J. Psychiatry 170(5), 542-549 (2013).

202. F. Kapczinski et al., "Peripheral biomarkers and illness activity in bipolar disorder," J. Psychiatry Res. 45(2), 156-161 (2011).

203. G. I. Papakostas et al., "Assessment of a multi-assay, serum-based biological diagnostic test for major depressive disorder: a pilot and replication study," Mol. Psychiatry 18(3), 332-339 (2013).

204. S. M. Wu and K. Hochedlinger, "Harnessing the potential of induced pluripotent stem cells for regenerative medicine (vol 13, pg 497, 2011)," Nat. Cell Biol. 13(6), 734-734 (2011).

205. K. J. Brennand et al., "Modeling psychiatric disorders at the cellular and network levels," Mol. Psychiatry 17(12), 1239-1253 (2012).

206. N. Pavillon et al., "Cell morphology and intracellular ionic homeostasis explored with a multimodal approach combining epifluorescence and digital holographic microscopy," J. Biophotonics 3(7), 432-436 (2010).

207. P. Campagnola, "Second harmonic generation imaging microscopy: applications to diseases diagnostics," Anal. Chem. 83(9), 3224-3231 (2011).

208. K. Fujita and N. I. Smith, "Label-free molecular imaging of living cells," Mol. Cells 26(6), 530-535 (2008).

209. N. Pavillon, A. J. Hobro, and N. I. Smith, "Cell optical density and molecular composition revealed by simultaneous multimodal labelfree imaging," Biophys. J. 105(5), 1123-1132 (2013).

Pierre Marquet is an MD-PhD who has obtained his board certification in adult psychiatry. Before his medical studies, he obtained a master's degree in physics from the Ecole Polytechnique Fédérale de Lausanne. He received a MD-PhD degree (MD-PhD fellowship from the Swiss National Science Foundation) in the field of digital holographic microscopy. He has been active for many years in the development of novel photonics-based techniques dedicated to address issues related to psychiatric neurosciences.

Biographies of the other authors are not available. 